\title{
The AIM2 inflammasome is a central regulator of intestinal homeostasis through the IL-18/IL-22/STAT3 pathway
}

\author{
Rojo A Ratsimandresy ${ }^{1}$, Mohanalaxmi Indramohan ${ }^{1}$, Andrea Dorfleutner ${ }^{1}$ and Christian Stehlik ${ }^{1,2}$
}

Inflammasomes are important for maintaining intestinal homeostasis, and dysbiosis contributes to the pathology of inflammatory bowel disease (IBD) and increases the risk for colorectal cancer. Inflammasome defects contribute to chronic intestinal inflammation and increase the susceptibility to colitis in mice. However, the inflammasome sensor absent in melanoma 2 (AIM2) protects against colorectal cancer in an inflammasome-independent manner through DNA-dependent protein kinase and Akt pathways. Yet, the roles of the AIM2 inflammasome in IBD and the early phases of colorectal cancer remain ill-defined. Here we show that the AIM2 inflammasome has a protective role in the intestine. During steady state, Aim2 deletion results in the loss of IL-18 secretion, suppression of the IL-22 binding protein (IL-22BP) in intestinal epithelial cells and consequent loss of the STAT3-dependent antimicrobial peptides (AMPs) Reg3 $\beta$ and Reg3 $\gamma$, which promotes dysbiosis-linked colitis. During dextran sulfate sodium-induced colitis, a dysfunctional IL-18/IL-22BP pathway in Aim2-/- mice promotes excessive IL-22 production and elevated STAT3 activation. Aim2 ${ }^{-1-}$ mice further exhibit sustained STAT3 and Akt activation during the resolution of colitis fueled by enhanced $R e g 3 b$ and Reg $3 g$ expression. This self-perpetuating mechanism promotes proliferation of intestinal crypt cells and likely contributes to the recently described increase in susceptibility of Aim2 $2^{-1-}$ mice to colorectal cancer. Collectively, our results demonstrate a central role for the AIM2 inflammasome in preventing dysbiosis and intestinal inflammation through regulation of the IL-18/IL-22BP/IL-22 and STAT3 pathway and expression of select AMPs.

Cellular \& Molecular Immunology (2017) 14, 127-142; doi:10.1038/cmi.2016.35; published online 15 August 2016

Keywords: antimicrobial peptides; IL-18; IL-22; IL-22BP; inflammasome; inflammatory bowel disease; microbiome; Reg3

\section{INTRODUCTION}

Inflammatory bowel disease (IBD) manifests most commonly as ulcerative colitis (UC) and Crohn's disease (CD), both of which are major health issues that severely impact quality of life and predispose patients to the development of colorectal cancer. CD and UC are chronic remittent inflammatory disorders that share several clinical features, including abdominal pain, diarrhea, rectal bleeding, malnutrition and fever. UC typically begins in the rectum, extends throughout the colon, with inflammation restricted to the mucosal surfaces, and is characterized by the presence of crypt abscesses. ${ }^{1}$ However, CD occurs predominantly in the small intestine or the proximal colon, with transmural inflammation characterized by granulomas, fissures and fistulas. ${ }^{2}$ Another distinction is the atypical Th2 or Th1/ Th17 cytokine profile characteristic of UC or CD patients, respectively. ${ }^{3}$ The pathology of IBD is still incompletely understood, but environmental and genetic risk factors contribute to IBD. The immune system has a key role in restricting and shaping intestinal microbial communities, and altered interactions between mucosal immune cells, intestinal epithelial cells (IECs) and the gut microbiome, due to a breached IEC barrier, contribute to the pathology of IBD. ${ }^{4}$

Inflammasomes are multi-protein platforms linking recognition of danger-associated molecular patterns and pathogenassociated molecular patterns to the activation of inflammatory caspase-1. Inflammasome sensors are pattern recognition

\footnotetext{
${ }^{1}$ Division of Rheumatology, Department of Medicine, Feinberg School of Medicine, Northwestern University, Chicago, IL 60611, USA and ${ }^{2}$ Robert H. Lurie Comprehensive Cancer Center, Interdepartmental Immunobiology Center and Skin Disease Research Center, Feinberg School of Medicine, Northwestern University, Chicago, IL 60611, USA

Correspondence: Dr A Dorfleutner or Dr C Stehlik, Division of Rheumatology, Department of Medicine, Feinberg School of Medicine, Northwestern University, 240 E Huron St, Chicago, IL 60611, USA.
}

E-mail: a-dorfleutner@northwestern.edu or c-stehlik@northwestern.edu

Received: 8 January 2016; Revised: 17 May 2016; Accepted: 17 May 2016 
receptors belonging to the Nod-like receptor (NLR) or AIM2like receptor (ALR) families. ${ }^{5,6}$ NLRs and ALRs contain either a PYRIN domain (PYD) or a caspase recruitment domain and cause the polymerization of the adaptor ASC and subsequent activation of caspase-1 by induced proximity. Active caspase- 1 is then responsible for the maturation and release of the cytokine substrates interleukin (IL)-1 $\beta$, IL-18 and a number of leaderless proteins as well as for the induction of pyroptotic cell death.

Several inflammasomes shape the mucosal immune response in a cell type-specific manner within the gastrointestinal tract. In particular, NLRP3 and NLRC4 sense a variety of enteric pathogens and pathobionts and initiate immune responses against these pathogens. ${ }^{7-9}$ In addition, several ASC-containing inflammasomes are involved in the maintenance of intestinal homeostasis and the prevention of dysbiosis; consequently, they are also involved in the pathology of IBD and colitis-linked colorectal cancer. ${ }^{3,4,10}$ Initial studies suggested that inflammasome activation and the production of downstream effectors is detrimental for colitis. The induction of colitis in mice caused elevated IL-18 in hematopoietic and epithelial tissues, and IL-18 transgenic mice displayed exacerbated disease, while neutralizing IL-18 ameliorated colitis. ${ }^{1-13}$ However, other studies have shown that $I l 18^{-1-}$ and $I l 18 r^{-1-}$ mice develop more severe colitis. ${ }^{14,15}$ Similarly, Casp $1 / 11^{-/-}$mice, or mice treated with caspase-1 inhibitor, show reduced IL- $1 \beta$, IL-18 and IFN- $\gamma$ and reduced colitis severity, ${ }^{16,17}$ while other studies revealed the opposite result caused by impaired tissue repair. ${ }^{18}$ In addition, deletion of the inflammasome sensor Nlrp3 or pharmacological inhibition of caspase-1 resulted in ameliorated dextran sulfate sodium (DSS)-induced colitis. ${ }^{19}$

In contrast, several recent studies have provided more detailed insights into inflammasome signaling in colitis, revealing that it is detrimental in the hematopoietic compartment, while it is protective in IECs, where IL-18 has a role in local tissue regeneration in response to injury. $\mathrm{Nlrp3}^{-1-}, \mathrm{Asc}^{-/-}$and Casp $1 / 11^{-1-}$ mice are all hyper-susceptible to DSS-induced colitis and are required for epithelial cell integrity. ${ }^{20-22}$ Loss of NLRP3 inflammasome activity and the consequent loss of IL-1 $\beta$ and IL-18 results in dissemination of commensal bacteria into the lamina propria, leukocyte infiltration and elevated chemokine production in the colon as well as increased incidence of colorectal cancer. ${ }^{20-23} \mathrm{Nlrp3}^{-1-}$ mice also develop dysbiosis and display reduced production of $\beta$-defensins, IL-10 and TGF- $\beta .^{22}$ In addition to NLRP3, the NLRP6 inflammasome in the hematopoietic and non-hematopoietic compartments also participates in colonic homeostasis. Nlrp $6^{-1-}$ mice show transferrable dysbiosis and increased susceptibility to DSS and colorectal cancer, suggesting that intestinal homeostasis requires the activation of multiple inflammasomes. ${ }^{15,24,25}$ Furthermore, NLRP12 may assemble an inflammasome as well as regulate $\mathrm{NF}-\mathrm{\kappa B}$, and Nlrp12-I- mice are more susceptible to colitis and colorectal cancer. ${ }^{26,27}$ Furthermore, Nlrc4 ${ }^{-1-}$ mice develop an increased colorectal tumor burden in a DSS model, although they do not show altered colitis. ${ }^{28}$ However, another study showed no effect on tumorigenesis. ${ }^{21}$
Recently, loss of Aim2 has been implicated in the development of DSS-induced colorectal cancer through an inflammasomeindependent mechanism, with no apparent effect on DSSinduced colitis. ${ }^{29,30}$ Non-hematopoietic AIM2 interacts with and inhibits the DNA-dependent protein kinase (DNA-PK), a PI3K-related kinase, which promotes Akt phosphorylation. ${ }^{30}$ In addition, AIM2 expression in intestinal stem cells prevents Wnt signaling, uncontrolled proliferation and dysbiosis. ${ }^{29}$

Here we show that imm $^{-/-}$mice are more susceptible to DSSinduced colitis. This phenotype is mediated by dysbiosis due to impaired IL-18 production by IECs, colitis-driven imbalanced ratios of IL-22/IL-22BP, excessive STAT3 signaling and dysregulation of the IL-18- and IL-22-dependent anti-microbial and pro-proliferative peptides of the Reg3 family.

\section{MATERIALS AND METHODS}

\section{Mice}

Aim2 $2^{-1-}$, Casp $1 / 11^{-1-}$ and WT mice were purchased from The Jackson Laboratory (Bar Harbor, ME, USA), and $\mathrm{Nlrp}^{-1-}$ and Asc $^{-1-}\left(\right.$ Pycard $\left.^{-1-}\right)$ mice were obtained from Dr Vishva M. Dixit at Genentech (San Francisco, CA, USA) Inc. ${ }^{31-36}$ Mice were generated or backcrossed onto the C57BL/6 strain for at least 10 generations, were bred and housed in a specific pathogen-free (SPF) animal facility at Northwestern University and provided chow and water ad libitum. All animal study protocols were approved by the Northwestern University Feinberg School of Medicine Institutional Animal Care and Use Committee (IACUC), and all experiments were performed on age- and gender-matched, randomly assigned 8-15-week-old mice.

\section{DSS-induced colitis}

Acute colitis was induced with 2-3\% (w/v) DSS (molecular mass 36-40 kDa; MP Biologicals, Santa Ana, CA, USA) dissolved in sterile, distilled water provided ad libitum on experimental days $1-5$, followed by normal drinking water until the end of the experiment on days 5,7 or 14 , as previously described. ${ }^{37}$ DSS was freshly dissolved every 2 days.

\section{Antibiotic treatment and fecal microbiota transplant}

For the antibiotics-only treatment, mice were equally treated with a combination of antibiotics for 14 days as previously described. ${ }^{15}$ Briefly, mice were given a combination of vancomycin $(1 \mathrm{~g} / \mathrm{l})$, ampicillin $(1 \mathrm{~g} / \mathrm{l})$, kanamycin $(1 \mathrm{~g} / \mathrm{l})$ and metronidazole $(1 \mathrm{~g} / \mathrm{l})$ (all from Sigma-Aldrich) for 3 weeks in their drinking water before the administration of DSS as described above. For fecal microbiota transplant (FMT), mice were treated with antibiotics as described above and then received fecal content from either WT or Aim2 $2^{-1-}$ mice. Briefly, donor animals were weight-, gender- and age-matched single-housed WT or Aim $2^{-/-}$mice. Mice were killed, and the cecal content was immediately diluted in sterile phosphate-buffered saline, filtered through a $500-\mu \mathrm{m}$ mesh and administered equally to each group of mice. Recipient mice were given $48 \mathrm{~h}$ to recover before DSS administration as described above. 


\section{Determination of clinical colitis scores}

Weight loss, stool consistency and any presence of occult or macroscopic blood were determined daily until mice were killed at days $0,5,7$ or 14 . Stool consistency and rectal bleeding were analyzed as previously described: ${ }^{37} 0$, normal stool; 1 , soft but still formed stool; 2, loose stool; 3, mostly liquid stool; 4, diarrhea; and 0 , negative hemoccult; 2, positive hemoccult; 4, blood traces visible in stool/rectal bleeding.

\section{Colon tissue analysis}

Mice were killed, and entire colons were isolated, measured and opened along the mesenteric border. Mesenteric fat and fecal material were removed, and colons were aseptically flushed several times with Hank's balanced salt solution (HBSS). The entire colon was then weighed, and samples were isolated in identical order for each mouse, starting from the distal portion of the colons.

Histology. One centimeter of colon was fixed in 10\% formalin overnight, embedded in paraffin, sectioned and stained with hematoxylin and eosin ( $\mathrm{H} \& \mathrm{E})$ and $\mathrm{Ki}-67$ at the Northwestern University Mouse Histology and Phenotyping Laboratory. The severity of colitis was scored histologically using two different parameters. An inflammation score: 0, no inflammation; 1, increased inflammatory cells noted above the muscularis mucosa only; 2, increased inflammatory cells involving the submucosa and above; 3 , increased inflammatory cells involving the muscularis and/or serosa. The percentage of ulceration was determined by assessing the relative extent of ulceration along the muscularis mucosa (expressed as percentage ulcerated mucosa).

Colon tissue explants. Biological triplicates of $0.5-\mathrm{cm}$ colon pieces per mouse were isolated; pre-incubated $30 \mathrm{~min}$ in HBSS supplemented with $100 \mu \mathrm{g} / \mathrm{ml}$ gentamicin; rinsed and cultured in complete RPMI 1640 medium supplemented with $10 \%$ fetal bovine serum (FBS), $100 \mu \mathrm{g} / \mathrm{ml}$ gentamicin, $1 \%$ penicillinstreptomycin and $10 \mathrm{mM}$ HEPES. Supernatants were collected after $17 \mathrm{~h}$, cleared by centrifugation at $12,000 \mathrm{~g}$ for $10 \mathrm{~min}$ at $4{ }^{\circ} \mathrm{C}$ and stored at $-80^{\circ} \mathrm{C}$ before determination of cytokine concentrations by enzyme-linked immunosorbent assay (ELISA). For cytokine measurements of select samples by ELISA, whole colon tissue was lysed in RIPA buffer containing protease and phosphatase inhibitors, and results were normalized to protein levels by BCA assay.

MPO activity. Myeloid peroxidase (MPO) was extracted from colon homogenates $(10 \% \mathrm{w} / \mathrm{v})$ with $0.5 \%$ hexadecyltrimethylammonium, and biological activity was measured according to the manufacturer's instructions (Enzo Life Sciences, Farmingdale, NY, USA).

\section{Cytokine measurement}

IL- $1 \beta$, TNF- $\alpha$, IL-6, IFN- $\gamma$ (all BD Biosciences, San Jose, CA, USA), IL-18 (R\&D Systems, Minneapolis, MN, USA), IL-22 (Affymetrix, Santa Clara, CA, USA) and IL-22BP (MyBiosource, San Diego, CA, USA) were determined by ELISA from clarified culture supernatants, colon tissue explants or colon lysates. ELISA results were colon weight-adjusted.

\section{Immunoblot}

Cells or whole colon tissues were lysed in RIPA buffer containing protease and phosphatase inhibitors. Lysates were separated by sodium dodecyl sulfate-polyacrylamide gel electrophoresis, transferred to polyvinylidene difluoride membranes and analyzed by immunoblot with the appropriate primary antibodies and horseradish peroxidase-conjugated secondary antibodies (whole donkey antibody to rabbit IgG (NA934V) and whole sheep antibody to mouse IgG (NXA931), both from GE Healthcare (Pittsburgh, PA, USA); ECL detection (ThermoFisher, Waltham, MA, USA) and digital image acquisition (Ultralum, Claremont, CA, USA)). The following antibodies were used: rabbit monoclonal antibody to phospho-STAT3 (Tyr705; clone D3A7), rabbit monoclonal antibody to total-STAT3 (clone 79D7), rabbit monoclonal antibody to phospho-Akt (Ser473; clone D9E) and rabbit monoclonal antibody to total Akt (clone C67E7) (Cell Signaling Technology, Danvers, MA, USA).

\section{Isolation of fecal DNA}

Immediately after euthanasia, fecal samples were collected from colons, frozen in liquid nitrogen and stored at $-80^{\circ} \mathrm{C}$ until further processing. DNA in colonic fecal samples was extracted using the QiaAmp DNA Stool Kit (Qiagen, Valencia, CA, USA) according to the manufacturer's instructions and quantified on a Synergy HT Multimode plate reader using the Take3 MicroVolume Plate attachment (BioTek, Winooski, VT, USA).

\section{Quantitative real-time PCR}

Total RNA was isolated and analyzed as described. ${ }^{35,36,38}$ Total RNA was isolated using TRIzol reagent (Invitrogen, ThermoFisher), treated with DNase I, reverse transcribed with iScript (Bio-Rad, Hercules, CA, USA) and analyzed by either predesigned FAM-labeled TaqMan real-time gene expression primer/ probes or the iTaq Universal SYBR Green real time PCR assays (Bio-Rad) on an ABI 7300 Real time PCR machine (Applied Biosystems, Carlsbad, CA, USA) and displayed as relative expression compared with $A c t b$. The following SYBR Green primer pairs for cytokines, HDPs and microbial 16S rDNA genes were used: Defcr5: 5'-AGGCTGATCCTATCCACAAAACAG-3', 5'-TGAAGAGCAGACCCTTCTTGGC-3'; ${ }^{39,40}$ Bd14: 5'-GTATT CCTCATCTTGTTCTTGG-3'， 5'-AAGTACAGCACACCGGCC AC-3 ${ }^{\prime} ;{ }^{40,41}$ Reg3b: 5'-GAGGCCTGGAGGACACCTCGT-3', 5'-T TGTCCCTTGTCCATGATGCTCTTC-3' ${ }^{\prime}{ }^{42}$ Reg $3 g$ : 5'-GCTCCT ATTGCTATGCCTTGTTTAG-3'， 5'-CATGGAGGACAGGAAG GAAGC-3'; ${ }^{\prime 40,43}$ Il6: 5'-TCCAATGCTCTCCTAACAGATAAG3', 5'-CAAGATGAATTGGATGGTCTTG-3'; ${ }^{\prime 2,44}$ Il22: 5' -TCCG AGGAGTCAGTGCTAAA-3', 5' -AGAACGTCTTCCAGGGTG AA-3 $3^{\prime}{ }^{40,44}$ Il22bp: 5' -TCAGCAGCAAAGACAGAAGAAAC-3', 5'-GTGTCTCCAGCCCAACTCTCA-3'; ${ }^{\prime 5}$ Gapdh: 5'-CTACAG CAACAGGGTGGTGG-3'， 5'-TATGGGGGTCTGGGATGG-3'; ${ }^{\prime}{ }^{\prime}$ Mouse Intestinal Bacteroides (MIB): 5'-CCAGCAGCCGCGGT AATA-3', 5'-CGCATTCCGCATACTTCTC-3'; ${ }^{40,46}$ Bacteroides: 5'-GAGAGGAAGGTCCCCCAC-3'， 5'-CGCTACTTGGCTGGT TCAG-3'; ${ }^{40,47}$ Clostridium leptum (cluster IV) 5'-CCTTCCG 
TGCCGSAGTTA-3'， 5'-GAATTAAACCACATACTCCACTGCT T- $3^{\prime} ;{ }^{40,48}$ Clostridium coccoides (cluster XIVa) 5'-ACTCCTACGG GAGGCAGC-3', 5'-GCTTCTTAGTCAGGTACCGTCAT-3' ${ }^{\prime}{ }^{40,46}$ Lactobacillus: 5'-AGCAGTAGGGAATCTTCCA-3', 5'-CACCGCT ACACATGGAG-3'; ${ }^{\prime 4,46}$ Bifidobacterium: $5^{\prime}$-CGGGTGAGTAAT GCGTGACC-3' 5' $^{\prime}$-TGATAGGACGCGACCCCA-3'; ${ }^{\prime 4,48}$ Bacillus: 5'-GCGGCGTGCCTAATACATGC-3', 5'-CTTCATCACTCACG CGGCGT-3'; ${ }^{\prime 2,47}$ Escherichia coli: 5'-CATGCCGCGTGTATGAA GAA-3', 5'-CGGGTAACGTCAATGAGCAAA-3'48; Prevotella: 5'-CCTWCGATGGATAGGGGTT-3', 5'-CACGCTACTTGGCT GGTTCAG-3';8 TM7: 5'-GAGAGGATGATCAGCCAG-3', 5'-G ACCTGACATCATCCCCTCCTTCC-3' ${ }^{\prime}{ }^{49}$ all bacteria: $5^{\prime}$-CGG TGAATACGTTCCCGG-3'， 5'-TACGGCTACCTTGTTACGACT $\mathrm{T}-3^{\prime}{ }^{48}$ The cycling parameters were as follows: $95^{\circ} \mathrm{C}$ for $15 \mathrm{~s}$ and then $49.5-55.0^{\circ} \mathrm{C}$ (depending on the annealing temperature of each primer set) for a total of 40 cycles. For each primer set, the quality of the melting curve was checked to ensure for the specificity of the amplification.

\section{Isolation of intestinal epithelial cells}

For $q P C R$. Cleaned colons were cut into $1-\mathrm{cm}$ pieces and cleared of debris by three additional washes in ice-cold HBSS. IECs were then isolated following treatment of colon pieces with $5 \mathrm{mM}$ EDTA and $2 \mathrm{mM}$ DTT in HBSS at $37^{\circ} \mathrm{C}$ for $30 \mathrm{~min}$. IECs fractions were washed in ice-cold HBSS, immediately lysed in TRIzol (Invitrogen, ThermoFisher) and stored at $-80^{\circ} \mathrm{C}$ until further processing. The purity of IEC fractions was assessed by flow cytometry (BD LSR II), and cells were $>85 \% \mathrm{EpCAM}^{+}$(rat anti-mouse EpCAM antibody, clone G8.8, eBioscience, San Diego, CA, USA) (data not shown).

For tissue culture. Organoids were obtained as previously described. ${ }^{50}$ Briefly, after cleaning, whole colons were cut into pieces, washed and incubated with $1 \mathrm{mg} / \mathrm{ml}$ collagenase $\mathrm{D}$ and $100 \mu \mathrm{g} / \mathrm{ml}$ dispase II for $1 \mathrm{~h}$, then organoids were purified by successive sedimentation steps in $2 \%(\mathrm{w} / \mathrm{v})$ sorbitol-HBSS. Organoids were then pelleted; re-suspended and cultured in DMEM medium supplemented with $2.5 \%$ FBS, $1 \%$ sodium pyruvate, $0.25 \mathrm{U} / \mathrm{ml}$ insulin, $100 \mathrm{U} / \mathrm{ml}$ penicillin, $30 \mu \mathrm{g} / \mathrm{ml}$ streptomycin and $100 \mu \mathrm{g} / \mathrm{ml}$ gentamicin. IECs were treated with recombinant IL-18 (500-1000 ng/ml; R\&D Systems), recombinant IL-22 (25-50 ng/ml; eBiosciences), recombinant Reg3 3 ( $2 \mu \mathrm{g} / \mathrm{ml}$; R\&D Systems), recombinant Reg3 $\gamma(1 \mu \mathrm{g} / \mathrm{ml}$; $\mathrm{R} \& \mathrm{D}$ Systems) and the neutralizing rat monoclonal anti-IL-18 antibody (10 $\mu \mathrm{g} / \mathrm{ml}$, clone \#93-10C; R\&D Systems).

\section{Immortalization of bone marrow-derived macrophages}

AMJ2-C11 alveolar macrophages infected with the J2 virus (ATCC CRL-2456) were used as the source for J2 virus production as previously described. ${ }^{51}$ Briefly, AMJ2-C11 cells were grown in DMEM, supplemented with $4 \mathrm{mM}$ L-glutamine, $1.5 \mathrm{~g} / \mathrm{l}$ sodium bicarbonate, $4.5 \mathrm{~g} / \mathrm{l}$ glucose, $5 \mathrm{mM}$ HEPES and $10 \%$ FBS. Cleared culture supernatants containing virus were collected from confluent cells after 3 days by centrifugation and $0.45 \mu \mathrm{m}$ filtration. These were then used to infect BMDMs that had been differentiated for 7 days in L929-conditioned medium $1: 1(\mathrm{v} / \mathrm{v})$ in $10-\mathrm{cm}$ Petri dishes in the presence of $3 \mu \mathrm{g} / \mathrm{ml}$
Polybrene (Sigma, St Louis, MO, USA) at $32^{\circ} \mathrm{C}$ for $16 \mathrm{~h}$, followed by culture in L929-conditioned medium. A homogenous immortalization of bone marrow-derived macrophages (iBMDM) population was obtained after 4-6 weeks.

\section{DNA transfection of iBMDM and IECs}

Lipofectamine 2000 transfection reagent (Invitrogen, ThermoFisher) was used for the transfection of iBMDM or IECs with fecal DNA or poly(dA:dT) as indicated, and IL-1 $\beta$ or IL-18 secretion was measured after $17 \mathrm{~h}$ by ELISA. Alternatively, cells were lysed in TRIzol (Invitrogen, ThermoFisher), and total RNA or protein was extracted following the manufacturer's instructions.

\section{Statistical analysis}

Graphs represent the mean \pm s.e.m. A standard two-tailed unpaired $t$-test and unpaired, non-parametric Mann-Whitney $U$-test were used for statistical analysis of groups. Values of $P<0.05$ were considered significant and are listed in the figure legends (Prism 5, GraphPad, La Jolla, CA, USA). The investigators were not blinded to the genotype of the mice/cells, but they were blinded to all sample and tissue analyses. Sample sizes were selected on the basis of preliminary results to ensure a power of $80 \%$ with $95 \%$ confidence between populations. All samples were analyzed in triplicate and were repeated at least three times, showing a representative result.

\section{RESULTS}

\section{AIM2 prevents intestinal dysbiosis}

Several inflammasomes maintain intestinal homeostasis, and their loss causes intestinal dysbiosis. ${ }^{3,4,10,15,20-27}$ We therefore investigated if AIM2 expression also contributes to a balanced intestinal microbiota and whether deletion of Aim2 would alter the composition of gut bacterial communities. We determined the relative presence of several bacterial strains from fecal DNA extracted from the colons of SPF WT and Aim $2^{-1-}$ mice by quantitative PCR utilizing specific bacterial $16 \mathrm{~S}$ rDNA primers. ${ }^{40,46-49}$ In Aim2 $2^{-/-}$mice, we detected increased populations of Prevotella, Bacteroides and mouse intestinal Bacteroides (MIB) within the phylum of Bacteroidetes compared with WT mice (Figure 1a). In particular, the increased abundance of Prevotella is reminiscent of $N l r p 6^{-1-}$ and $A s c^{-/-}$mice. ${ }^{15}$ However, contrary to $\mathrm{Nlrp}^{-1-}$ and $A s \mathrm{C}^{-1-}$ mice, which display an increased number of members of the phylum TM $7,{ }^{15}$ Aim $2^{-1-}$ mice showed reduced TM7 (Figure 1b). Hence, the various inflammasomes appear to involve distinct mechanisms of regulating the composition of the intestinal microbiota. We did not detect significant differences in the abundance of key Gram-positive or Gram-negative groups, including Lactobacillus, Bacillus, Eubacteria and Clostridium from the phylum Firmicutes; Bifidobacterium from the phylum Actinobacteria or Escherichia from the phylum Proteobacteria (Figure 1c). Overall, we found that AIM2 contributed to the homeostasis of the intestinal microbiota, and its loss caused the dysbiosis of select intestinal microbiota species. 

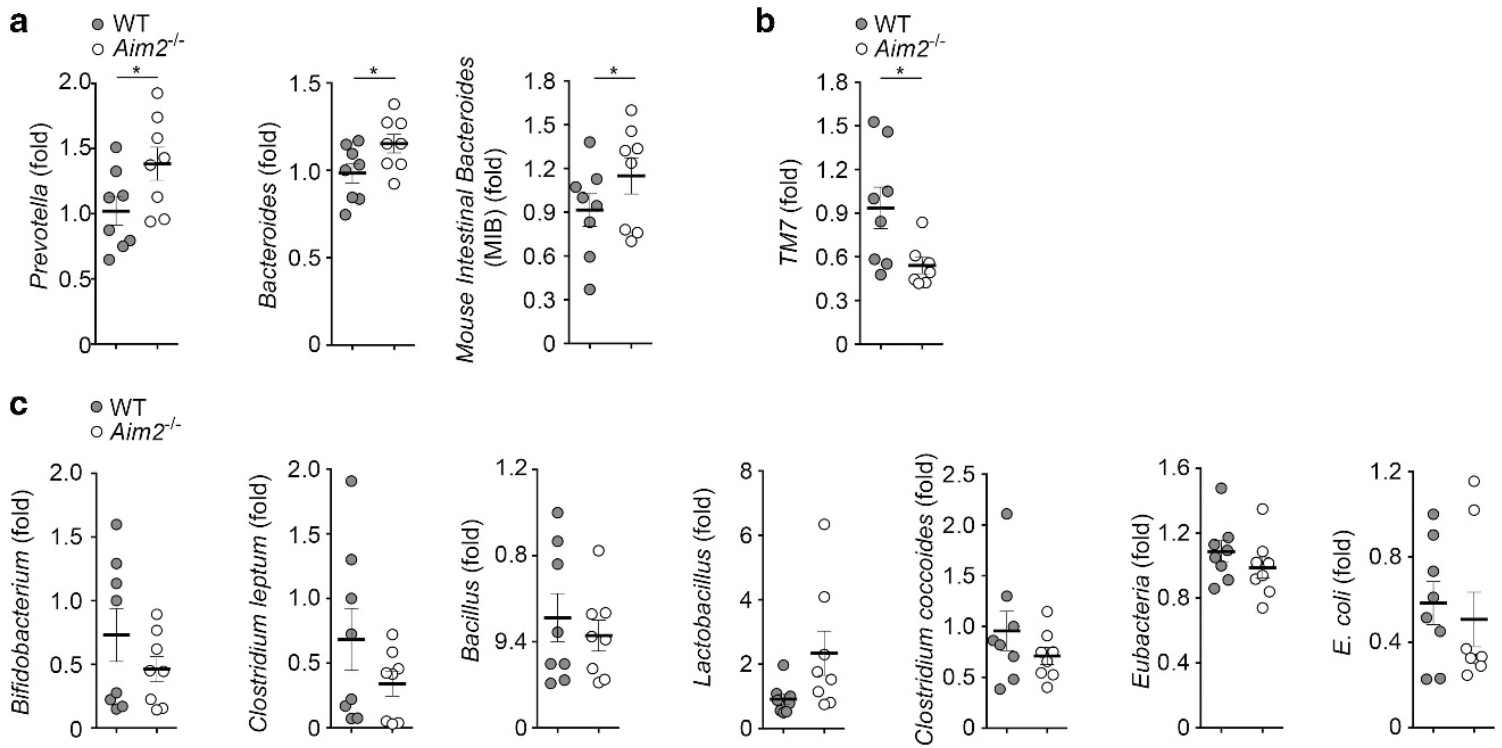

Figure 1 Loss of Aim2 causes intestinal dysbiosis. (a-c) DNA isolated from colonic fecal samples from naive WT and Aim2-1- mice was tested by real-time PCR for the indicated key microbial species $(n=6-8)$. The data are presented as fold induction compared with a randomly chosen naïve WT mouse. Error bars represent \pm s.e.m.; ${ }^{*} P<0.05$.

\section{AIM2 protects from acute DSS-induced colitis and prevents intestinal damage}

Compelling evidence supports the notion that alterations in the intestinal microbiota are linked to the development of IBD and exacerbate IBD pathology, which subsequently changes the intestinal milieu and promotes altered mucosal immunity in genetically susceptible individuals. Consequently, the intestinal microbiota of IBD patients differ from that of healthy people and show a $30-50 \%$ reduced commensal biodiversity. ${ }^{52}$ In addition, several inflammasomes have been linked to the immune response during experimental colitis. We therefore investigated if Aim2 $2^{-1-}$ mice were more susceptible to colitis. Oral administration of DSS in the drinking water causes apoptosis of IECs within basal crypts and affects the integrity of the mucosal barrier, which promotes the subsequent mucosal influx of commensal microbes, intestinal inflammation and eventually colitis. ${ }^{53}$ We orally administered the commonly used dose of 3\% DSS in drinking water to age- and gender-matched WT and Aim $2^{-1-}$ mice for 5 days and assessed the early stage of the disease starting at day 5 , the peak of the disease starting at day 7 and the resolution phase of acute colitis through day 14 (Figure 2a). Starting at day $7, A i m 2^{-1-}$ mice suffered more severe weight loss compared with WT mice. While WT mice lost up to $11.5 \%$ body weight, peaking at day $8, A i m 2^{-1-}$ mice lost up to $21.9 \%$ body weight, and their recovery began a day later than WT mice (Figure 2b). However, the recovery curve of Aim2 $2^{-1-}$ mice was steeper than that of WT mice, indicating that a loss of AIM2 may accelerate the resolution of inflammation and promote tissue repair. The increased susceptibility of Aim2 $2^{-1-}$ mice is reminiscent of other inflammasome componentdeficient mice, including $\mathrm{Nlrp}^{-1-}$ and $\mathrm{Asc}^{-1-}$, to $2 \%$ DSSinduced colitis (Figure 2c). Aim $2^{-1-}$ mice also displayed severe diarrhea compared with WT mice, as indicated by worse stool consistency (Figure 2d) and rectal bleeding scores (Figure 2e). Colon-length reduction is a gross assessment of the severity of DSS-induced colitis. ${ }^{54}$ In agreement with the more severe colitis symptoms, Aim $2^{-1-}$ mice also displayed a $9 \%$ shorter colon than WT mice at the peak of the disease on day 7 (Figures $2 \mathrm{f}$ and $\mathrm{g}$ ); however, colon length (Figure $2 \mathrm{~g}$ ) and thickness (Figure $2 \mathrm{~h}$ ) were not significantly different upon resolution of colitis at day 14. We verified these clinical parameters by histological examination of paraffin-embedded colon sections. DSS administration resulted in notable histopathological changes in $\mathrm{H} \& \mathrm{E}$-stained sections of colon tissue at the peak of the disease 7 days after DSS treatment, whereas the changes were less pronounced during recovery at day 14 (Figure 2i). DSS treatment caused extensive loss of crypts and more severe ulceration in the colons of Aim $2^{-1-}$ mice compared with WT mice. Aim $2^{-1-}$ mice also showed highly reduced goblet cells and increased severity of inflammation, as determined by the presence of infiltrating leukocytes, which often reached the transmural levels in Aim2 $2^{-1-}$ mice during acute colitis. Semi-quantitative scoring of these histological parameters confirmed that the severity of colitis was significantly higher in Aim $2^{-/-}$mice compared with WT mice during acute colitis (Figure 2j). In addition, Aim $2^{-1-}$ mice also displayed a greater extent of ulceration and necrotic lesions along the muscularis mucosa compared with WT mice during acute colitis, but not after colitis recovery, providing additional evidence for accelerated intestinal repair in Aim $2^{-1-}$ mice (Figure 2k). Increased leukocytic infiltration in the colons of Aim2 $2^{-1-}$ mice in response to DSS administration was further evident by higher MPO activity in the colons of Aim2 $2^{-1-}$ mice at the acute phase (Figure 21). 

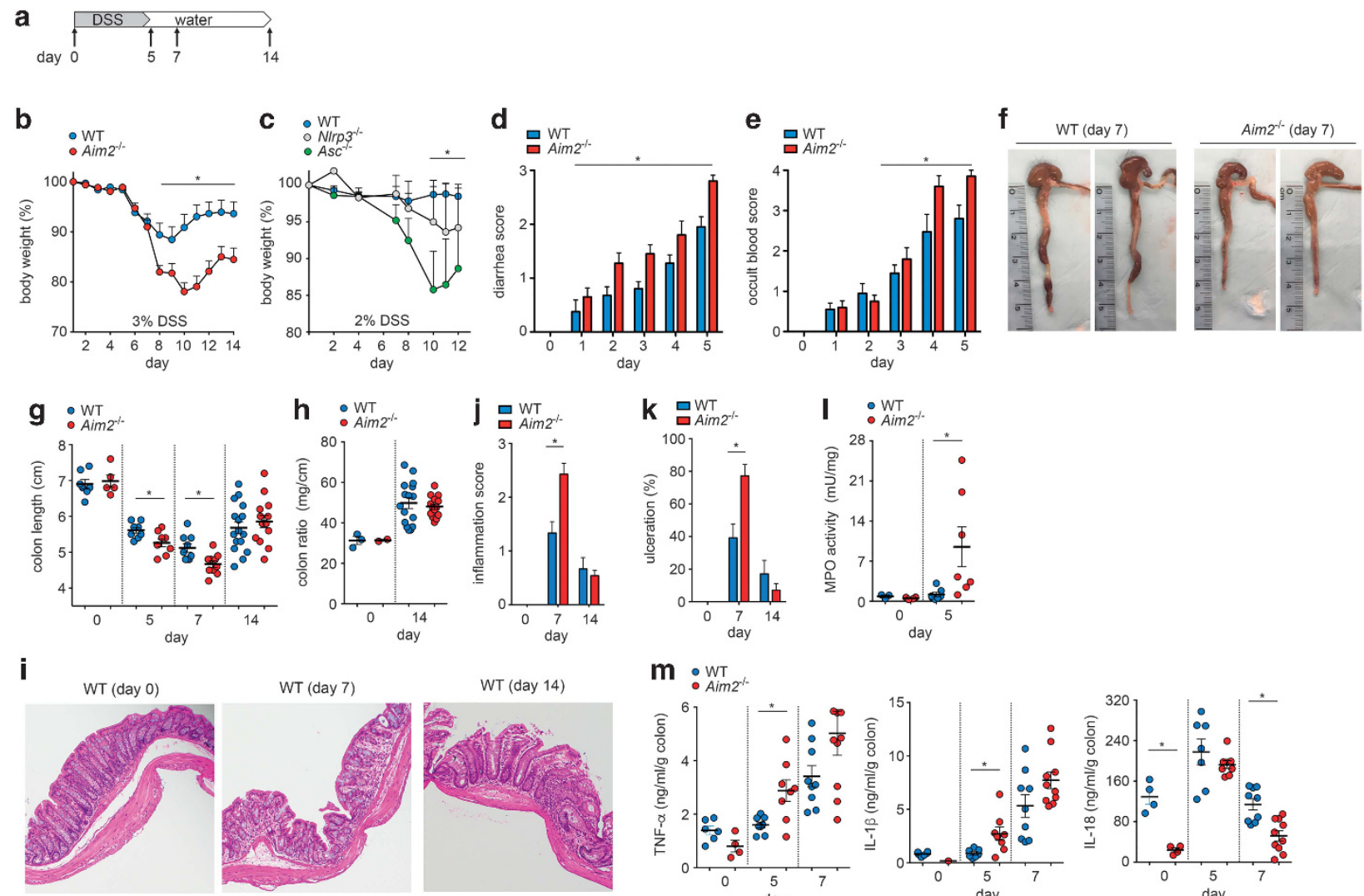

WT (day 7)

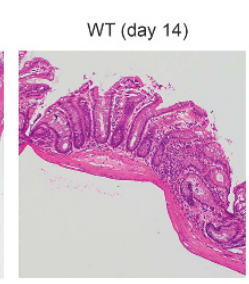

m: ${ }_{\text {Aim2 }}$
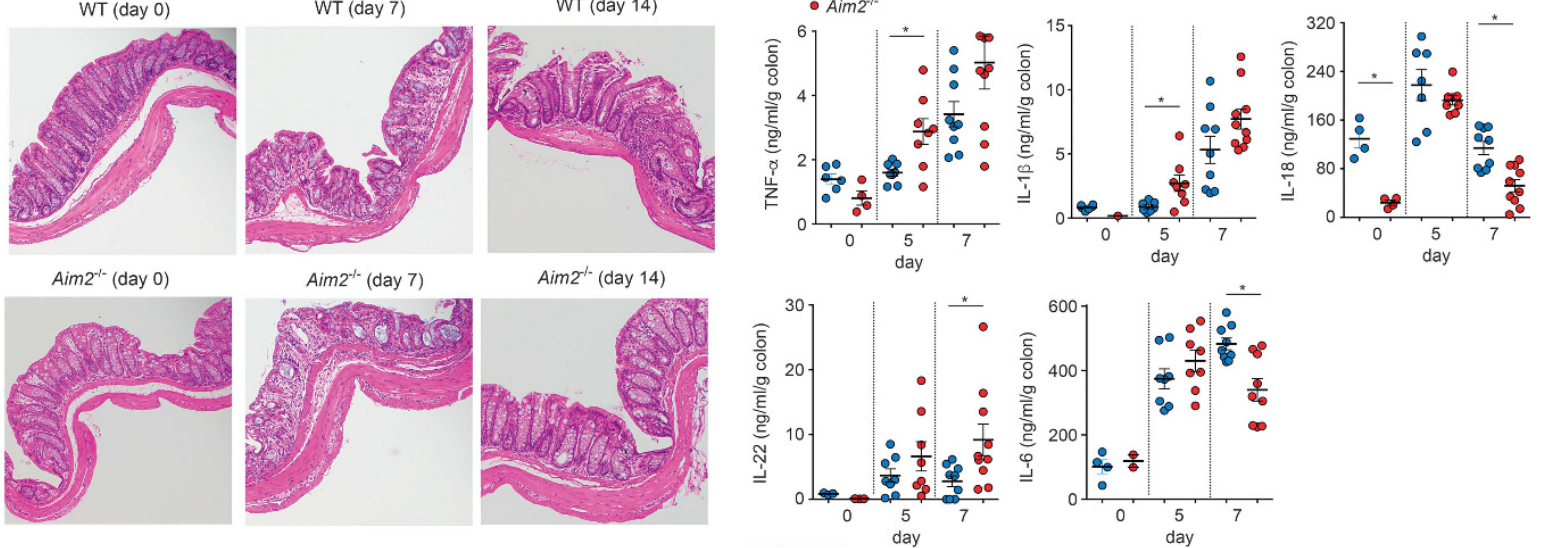

Figure 2 Aim2 $^{-1-}$ mice are more susceptible to DSS-induced colitis. (a) Timeline of DSS administration and analysis of colitis. WT and Aim2 -1 - mice were fed $3 \%$ DSS in drinking water for 5 days, followed by regular drinking water until euthanasia. Because of the increased susceptibility to colitis, N/rp3 ${ }^{-1-}, \mathrm{Asc}^{-1-}$ and WT mice were given 2\% DSS. All samples were harvested at day 0 (controls), day 5 (onset of colitis), day 7 (peak of colitis) and day 14 (resolution of colitis) following DSS administration. (b, c) Body weight loss was monitored during the progression of colitis induced with (b) $3 \%$ and (c) $2 \%$ DSS (b: $n=10, \mathbf{c}: n=5$ ). (d) Diarrhea score and (e) gross/occult rectal bleeding were evaluated daily $(n=10)$. (f, g) Colon lengths were measured at necropsy (f) representative pictures, (g) length at day 0 ( $n=6-7)$, day 5 $(n=8)$, day $7(n=10)$ and day $14(n=15-16)$ after DSS administration. (h) Colon thickness was measured at day 14 as a gross assessment of the resolution of colitis $(n=15-16)$. (i) Colon tissues harvested at day 0 , day 7 and day 14 were stained with H\&E to assess the severity of colitis at different phases, with representative images shown. (j, k) Semi-quantitative scoring of histopathology $(n=6-7)$ for (j) inflammation and (k) ulceration, (I) Myeloperoxidase (MPO) activity was determined and normalized per mg colon tissue at day 0 and day 5 of colitis $(n=6-7)$. (m) Washed colon tissue explants were weighed and cultured ex vivo for $17 \mathrm{~h}$, and cleared colon culture supernatants from colon biopsies normalized to weight at day $0(n=2-6)$, day $5(n=6-8)$ and day $7(n=9-11)$ were analyzed by ELISA for TNF- $\alpha$, IL-1 $\beta$, IL-6, IL-18 and IL-22. Error bars represent \pm s.e.m.; ${ }^{*} P<0.05$.

Overall, these results indicate that AIM2 is an important component that contributes to the protection from acute DSS-induced colitis and that AIM2 deficiency promotes an inflammatory intestinal environment. Imbalanced cytokine responses have a central role in the pathology of IBD. ${ }^{55}$ We therefore analyzed cytokines secreted from colon tissue explants ex vivo during the acute phase of disease at days 5 and 7 after DSS administration. In agreement with more severe colitis, we detected increased TNF- $\alpha$, IL- $1 \beta$ and IL-22 levels in colon tissue explants of $\mathrm{Aim}^{-1-}$ mice (Figure 2m), and these results were verified by direct analysis of colon tissue lysates (data not shown). However, we observed reduced IL-6 in Aim $2^{-I-}$ mice during the peak of the disease at day 7, which is in agreement with a protective role for IL-6 in IECs during colitis (Figure 2m). ${ }^{56-58}$ In contrast, IL-18 was reduced at all time points (Figure $2 \mathrm{~m}$ ), in agreement with impaired inflammasome activation reminiscent of $\mathrm{Asc}^{-/-}$ mice. $^{20,21,59}$ 
Dysbiosis in Aim2 $2^{-1-}$ mice is responsible for the heightened susceptibility to DSS-induced colitis

To investigate a causal connection between dysbiosis and the increased susceptibility to colitis in Aim $2^{-/-}$mice, we treated mice with an antibiotic cocktail $(\mathrm{ABx})$ to eliminate the microbiota for 2 weeks, followed by induction of DSS-induced colitis (Figure 3a). After treatment with $\mathrm{ABx}$, both WT and Aim2 $2^{-/-}$ mice failed to develop significant DSS-induced colitis, as indicated by equal but modest body weight loss, even at day 9 after DSS administration, which is past the peak of colitis (Figure 3b). Consequently, there were no differences in colon length at day 9 (Figures $3 \mathrm{c}$ and $\mathrm{d}$ ). To directly demonstrate that the altered microbiota in Aim $2^{-1-}$ mice was responsible for the susceptibility to colitis, we randomly assigned WT mice to two groups that were subjected to $\mathrm{ABx}$ treatment, after which these mice did not show any differences in body weight (Figure 3e). We next isolated and pooled fecal material from the colons of three WT or Aim2 $2^{-1-}$ donor mice that had not been treated with $\mathrm{ABx}$ and performed a FMT by gavage at days 1 and 3 into $\mathrm{ABx}$-treated WT recipient mice (Figure 3a). ${ }^{60}$ Fecal material from $A i m 2^{-1-}$ mice caused more severe colitis than fecal material from WT mice as determined by body weight (Figure 3f) and significantly shortened colon length on day 7 after DSS administration (Figures $3 g$ and $h$ ). These results indicate that dysbiosis is responsible for the susceptibility to

a
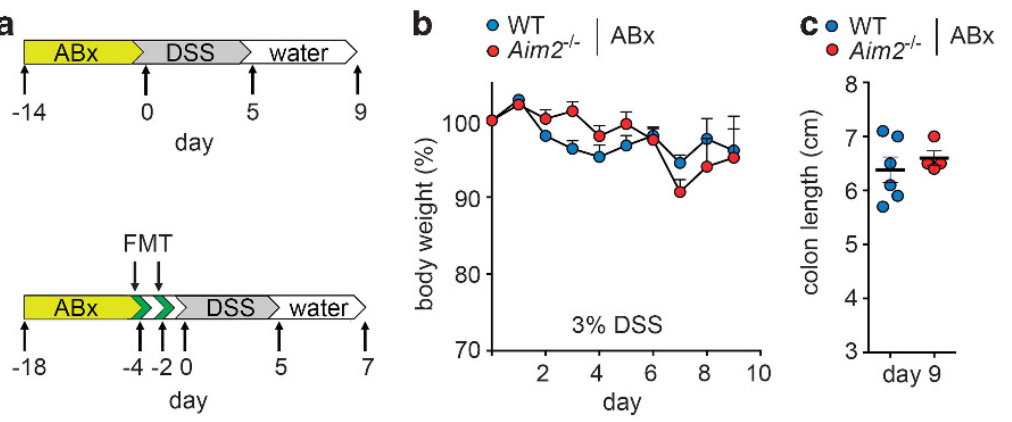

e

f

- WT FMT recipients
- Aim2 $2^{-1-}$ FMT recipients

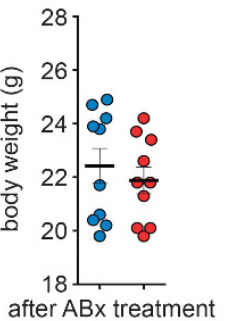

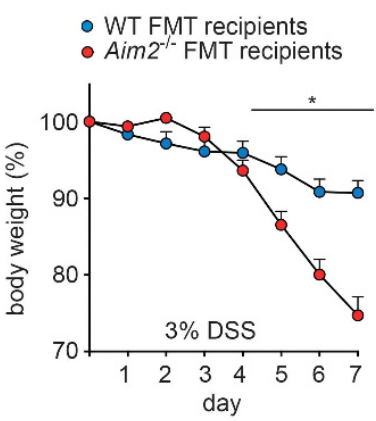

g

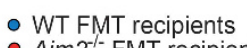

- Aim2 $^{-/-}$FMT recipients

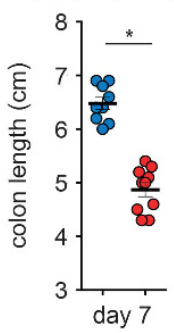

DSS-induced colitis in $A i m 2^{-1-}$ mice, as fecal material of Aim $2^{-1-}$ mice alone was sufficient to recapitulate the colitis prone phenotype observed in Aim $2^{-1-}$ mice.

The AIM2 inflammasome is functional in IECs and impacts colonic IL-22BP production

AIM2 assembles a DNA-sensing inflammasome in macrophages, which is essential for host defense against viral and bacterial pathogens. ${ }^{33,35,61,62}$ We therefore investigated whether genomic DNA from the commensal microbiota also induces AIM2 inflammasome activation in IECs. As gut bacteria make up the majority of feces, we isolated fecal genomic DNA (fDNA) from intestinal fecal samples. As expected, transfection of fDNA into immortalized WT iBMDM caused the release of IL-1 $\beta$ and IL-18, which was completely abrogated in Aim $2^{-/-}$iBMDM and matched the loss of response observed in $\mathrm{Asc}^{-1-}$ iBMDM and Casp1/11 ${ }^{-1-}$ iBMDM (Figures $4 \mathrm{a}$ and $\mathrm{b}$ ). We next examined, whether IECs respond to fDNA with IL-18 release, as epithelial cells are not a major source of IL-1 $\beta$. Transfection of fDNA into WT IECs resulted in IL-18 release, although this was less than that observed in macrophages. Importantly, IL-18 release was significantly reduced in IECs isolated from $A i m 2^{-1-}$ mice (Figure 4c), indicating that IECs assemble an AIM2 inflammasome that senses fDNA.
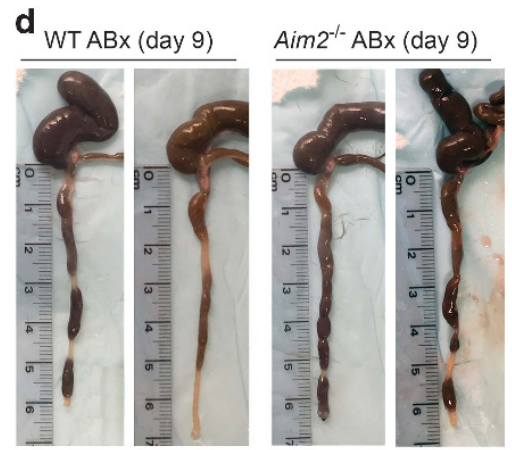

h

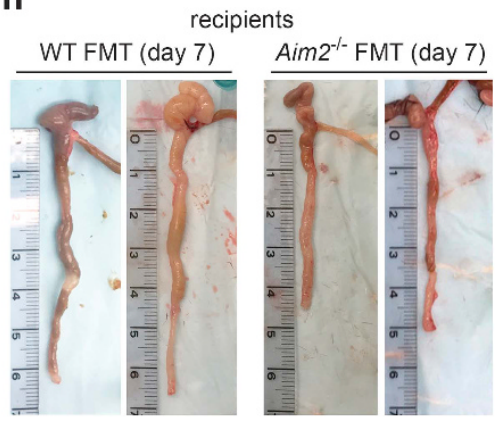

Figure 3 Transferrable dysbiosis in Aim2-1- mice is responsible for the susceptibility to DSS-induced colitis. (a) Timeline of ABx, FMT and DSS administration and analysis of colitis. Mice were fed 3\% DSS in drinking water for 5 days, followed by regular drinking water until euthanasia at day 7 or 9. (b-d) Mice were treated for 3 weeks with an antibiotics cocktail, followed by administration of 3\% DSS. (b) Body weight loss was monitored daily, and (c) colon length was determined at necropsy on day 9 after DSS administration. (d) Representative images are shown. (e-h) WT mice were treated with antibiotics as above, then (e) body weight was determined before re-colonization with either WT or Aim2 ${ }^{-1-}$ fecal microbiota by oral gavage before colitis induction with 3\% DSS. (f) Body weight was determined daily, and $(\mathbf{g}, \mathbf{h})$ colon length was determined at necropsy on day 7 after DSS administration. (h) Representative images are shown. The results represent individual mice $(n=7-10)$. Error bars represent \pm s.e.m.; ${ }^{*} P<0.05$. 

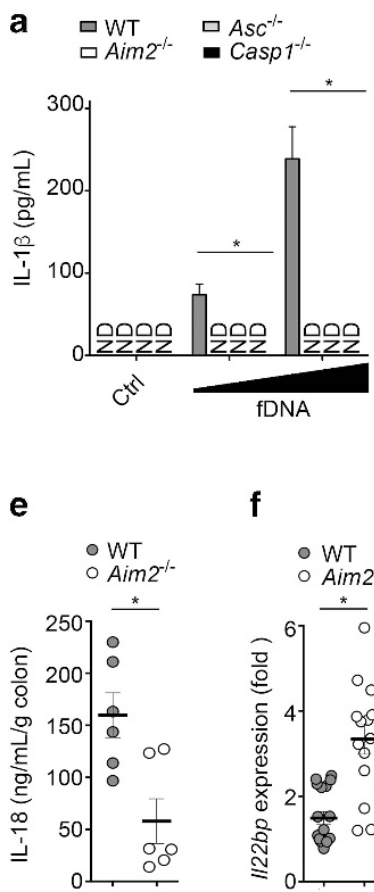

f
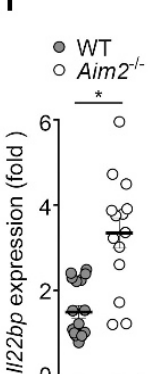

b

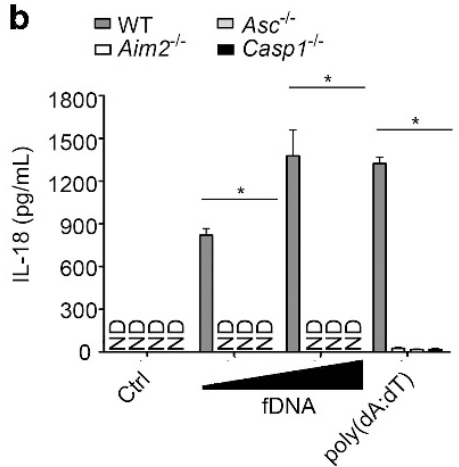

g

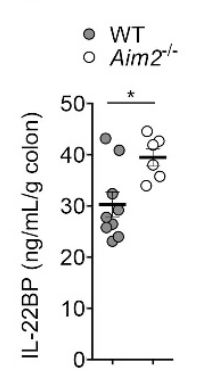

C

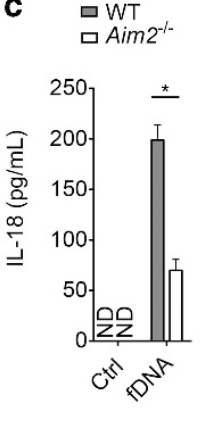

h

므 Ctrl
ㄷim2

므 $\operatorname{Aim}^{-1-}$

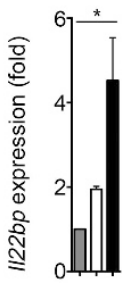

i

므 Ctrl

므 $4 \mathrm{hr}$
$8 \mathrm{hr}$

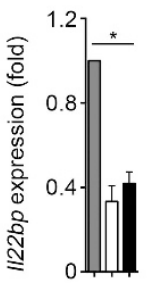

d $\circ W T$

$\circ \operatorname{Aim} 2^{-/-}$

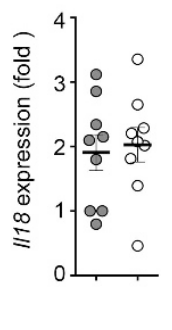

믄

ㅁ isotype Ctrl Ab

- anti-IL-18 Ab

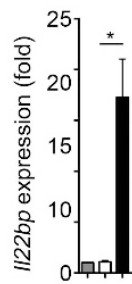

Figure 4 The AIM2 inflammasome senses fecal microbiota DNA and responds with IL-18 release to regulate IL-22BP. (a, b) Immortalized bone marrow-derived macrophages (iBMDM) were transfected for $17 \mathrm{~h}$ with (a) 0.25 or $0.5 \mathrm{ug} / \mathrm{ml}$ fecal DNA (fDNA) and (b) with 1 or 2 ug/ $\mathrm{ml}$ fDNA, or $1 \mathrm{ug} / \mathrm{ml}$ poly(dA:dT), and cleared culture supernatants were analyzed for (a) IL-1 $\beta$ and (b) IL-18. (c) IECs were seeded equally per genotype and transfected with $4 \mathrm{ug} / \mathrm{ml}$ fDNA for $17 \mathrm{~h}$, and IL-18 was determined as above. (d, f) IECs from naïve mice were analyzed by real-time PCR for the expression of (d) //18 and (f) $/ / 22 b p(n=9-14)$. The data are presented as fold induction compared to a randomly chosen WT mouse. (e, g) Spontaneous release of (e) IL-18 and (g) IL-22BP was determined from colon tissue explants of naïve WT and Aim2 $2^{-1-}$ mice after culture for $17 \mathrm{~h}(n=6-9)$. (h) IECs from naïve WT, Aim2 ${ }^{-1-}$ and Casp $1 / 11^{-1-}$ mice were analyzed by real-time PCR for the expression of $1 / 22 \mathrm{bp}$. (i, j) WT IECs were treated with (i) recombinant IL-18 (1 ug/ml), (j) anti-IL-18 or isotype control antibodies (20 ug/ml), and //22bp expression was analyzed by real-time PCR. Error bars represent \pm s.e.m.; ${ }^{*}<0.05$.

IL-18 has a key role in maintaining intestinal homeostasis. ${ }^{3}$ Specifically, inflammasome-mediated IL-18 suppresses the transcription of Il22bp in $\mathrm{CD}_{11 c^{+}}$dendritic cells (DCs). Il22bp encodes the soluble IL-22 receptor A2, also known as IL-22BP, which is produced by myeloid cells as well as IECs. ${ }^{63}$ IL-22BP lacks a transmembrane and intracellular domain and therefore antagonizes IL-22 signaling, which also has a key role in intestinal inflammation and repair. ${ }^{64}$ IL-22 is produced by a heterogeneous population of lymphocytes, including subsets of Th17, Th22 and ILC3 cells, but not by IECs. ${ }^{65,66}$ Because IECs responded to fecal microbiota DNA with IL-18 production, we investigated if this response could directly affect IL-22BP. We therefore isolated IECs from naive mice and determined the expression of $I l 18$ and Il22bp by qPCR. Similar to macrophages, we found that $I l 18$ was not regulated at the transcriptional level in IECs of naive mice (Figure 4d). However, IL-18 production from colon tissue explants was significantly reduced in naive Aim2 $2^{-1-}$ mice (Figure 4e), indicating that it is regulated by AIM2 inflammasome-mediated proteolytic maturation and secretion. In accordance with the impaired IL-18 production, IECs from Aim2 $2^{-/-}$mice expressed significantly more Il22bp than IECs isolated from WT mice (Figure 4f). In agreement with our qPCR results in IECs, colon tissue from Aim2 $2^{-/-}$mice also produced significantly elevated levels of IL-22BP (Figure 4g).
Additional support for the involvement of the AIM2 inflammasome in regulating Il22bp came from the observation that Casp $1 / 11^{-1-}$ IECs also showed elevated Il22bp expression (Figure $4 \mathrm{~h}$ ). To directly verify that Il22bp expression was dependent on IL-18, we treated IECs with recombinant IL-18 for 4 and $8 \mathrm{~h}$, which resulted in a 60\% reduction in Il22bp expression (Figure 4i). Conversely, incubating IECs for $8 \mathrm{~h}$ in the presence of an anti-IL-18 neutralizing antibody significantly increased Il22bp expression, while an isotype control antibody had no effect (Figure 4j). Our results indicate that AIM2 is necessary to promote IL-18 secretion from IECs, which in turn directly regulates the expression of Il22bp, and the loss of AIM2 likely contributes to an imbalanced IL-22BP/IL-22 ratio.

\section{AIM2 controls anti-microbial peptide production in IECs}

A key function of IECs is the production of AMPs to shape and maintain a healthy luminal microbiota, and IL-18 and IL-22 have a key role in this process. ${ }^{67-69}$ Because we observed an altered intestinal microbiota in Aim $2^{-1-}$ mice, and because AMP expression and the antimicrobial barrier are impaired in murine colitis and human $\mathrm{IBD},{ }^{70}$ we determined the expression of several key AMPs in IECs isolated from naive mice. Expression of the $\alpha$-defensin cryptdin-5 (Defcr5), the $\beta$-defensin Bd14 and the C-type lectin regenerating islet-derived (Reg) 2 were 
significantly elevated in Aim2-/- IECs (Figure 5a). In contrast, the expression of Reg $3 b$ and Reg3g was completely impaired in Aim $2^{-l-}$ IECs (Figure 5b). In addition, the expression of several other AMPs, including Reg1, Bd3 and the cathelicidin Cramp, was unchanged in Aim2 $2^{-/-}$IECs (Figure 5c). Our results indicate that AIM2 is necessary for the appropriate expression of select AMPs, specifically members of the Reg3 family. Defective AMP expression in Aim2 $2^{-1-}$ IECs was likely responsible for the observed dysbiosis in naive $A i m 2^{-1-}$ mice and consequently the heightened susceptibility of $A \mathrm{im}^{-1-}$ mice to colitis. Therefore, AIM2 has a key role in maintaining intestinal homeostasis.

\section{AIM2 controls anti-microbial peptide production and resolution of intestinal inflammation during DSS-induced colitis}

Because AMP expression is altered during acute colitis ${ }^{70}$ and the loss of Aim2 affects AMP expression in naive mice, we investigated AMP expression during DSS-induced colitis. We isolated IECs from WT and Aim2 $2^{-1-}$ mice at the acute and repair phases of colitis 5 and 14 days post DSS administration, respectively, and investigated the production of epithelial AMPs that were altered in naive Aim2 ${ }^{-1-}$ IECs. Contrary to naive Aim2 $2^{-1-}$ IECs, where the expression of Reg $3 b$ and Reg3g was impaired, we observed that both AMPs were significantly elevated in Aim2 ${ }^{-/-}$ IECs at both time points during DSS-induced colitis, whereas WT IECs were not significantly altered (Figure 6a). In contrast, expression of Defcr5 was strongly induced during colitis. However, Aim $2^{-1-}$ IECs showed reduced Defcr 5 expression compared with WT IECs 5 days post DSS administration, but they normalized during repair at day 14 (Figure 6b). Bd14 expression was elevated during acute colitis and was further elevated in Aim2 $2^{-1-}$ IECs, but it was downregulated during repair 14 days after DSS administration (Figure 6c). Reg3b and Reg3g were the only AMPs detected that were consistently upregulated in Aim2 $2^{-/-}$IECs during acute colitis and repair. Because their expression has been linked to IL-22, ${ }^{71}$ which was significantly increased in Aim2 ${ }^{-1-}$ mice during acute colitis (Figure 2m), it is likely that Reg3b and Reg3g expression also depends on IL-18/IL-22BP.

We therefore investigated Il22bp expression in IECs during colitis. Il22bp expression is downregulated in DCs during acute colitis, ${ }^{63}$ and we observed a comparable Il22bp expression pattern in IECs during acute colitis (Figure 6d). As we observed above in naive IECs (Figures $4 \mathrm{f}$ and g), Il22bp expression was also elevated in Aim2 $2^{-l-}$ IECs during colitis compared with WT IECs (Figure 6d). The reduced Il22bp expression on day 5 compared with day 0 correlated with elevated IL-22 (Figure 2m). Consistent with the increased Il22bp expression during the resolution phase at day 14 after DSS administration, IL-22 was reduced in Aim2 ${ }^{-1-}$ mice (Figure 6e). In addition, TNF- $\alpha$, IL-1 $\beta$ and IFN- $\gamma$ levels were also all reduced in Aim2 ${ }^{-/-}$mice 14 days after DSS administration (Figure 6e), suggesting a more rapid resolution of intestinal inflammation in $A i m 2^{-l-}$ mice.

\section{AIM2 regulates tissue repair through the IL-18/IL-22/STAT3/ Reg3 axis}

IL-22 can have opposing roles during colitis by exacerbating acute inflammation and antimicrobial immunity but also enhancing epithelial cell proliferation and tissue repair, thus contributing to the development of tumors. ${ }^{64}$ However, the role of IL-22, which signals primarily through STAT3, has not yet been investigated in the context of intestinal inflammation in Aim $2^{-1-}$ mice. We therefore isolated IECs from WT and Aim $2^{-1-}$ mice at the acute and repair phases of colitis at days 5 and 14 after DSS administration, respectively. Aim2 $2^{-1-}$ colons revealed enhanced phosphorylation of STAT3 on $\mathrm{Tyr}^{705}$ both at days 5 and 14 after DSS treatment, while total STAT3 was not different between WT and Aim2 $2^{-1-}$ colons (Figures 7a and b). These results suggest that IL-22 may be central to the elevated STAT3 signaling in Aim $2^{-1-}$ IECs during the acute phase of colitis. However, because IL-22 was reduced in Aim2 $2^{-1-}$ colons at day 14, additional factors may contribute to elevated STAT3 activation during intestinal repair. To investigate the cellular mechanisms associated with the differential regulation of STAT3 in IECs, we isolated WT IECs and treated organoids with recombinant IL-18 or IL-22. While IL-18 at any concentration failed to induce phosphorylation of STAT3, recombinant IL-22 induced phosphorylation of STAT3 in IECs (Figures 7c and d and data not shown), confirming that IL-22 alone elicits a direct effect on STAT3 signaling in IECs. In addition, an anti-IL-18 blocking antibody did not affect IL-22-mediated STAT3 phosphorylation (Figure 7d). To detect STAT3 phosphorylation in vitro, we treated cells for only $45 \mathrm{~min}$. During this short
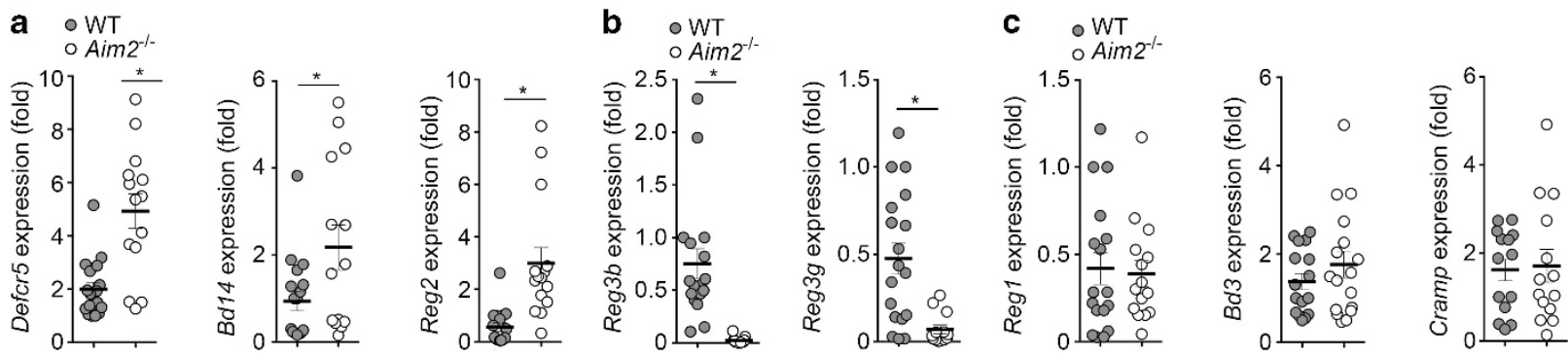

Figure 5 Aim2 deficiency impairs antimicrobial peptide production in IECs. (a-c) IECs from naive WT and Aim2 $2^{-1-}$ mice were analyzed by real-time PCR for the expression of (a) Defcr5, Bd14 and Reg2, (b) Reg3b and Reg3g and (c) Reg1, Bd3 and Cramp ( $n=14-16$ ). The data are presented as fold induction compared with a randomly chosen WT mouse. Error bars represent \pm s.e.m.; ${ }^{*} P<0.05$. 

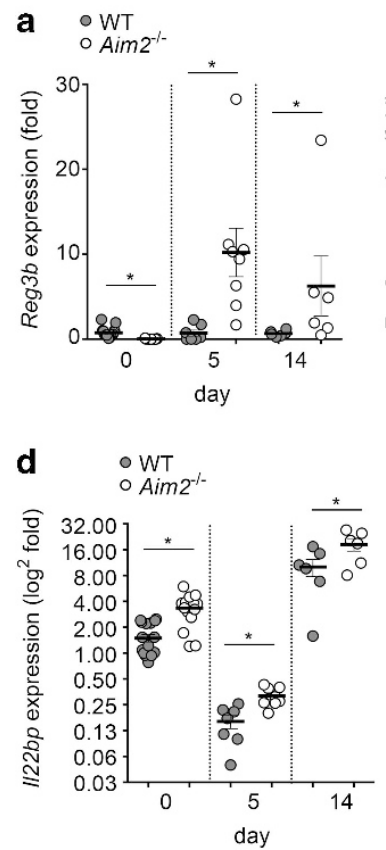

b

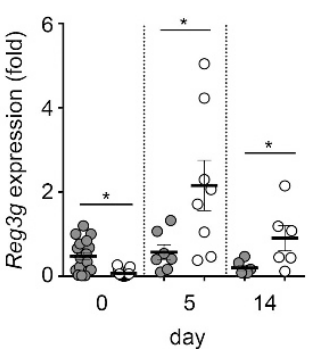

e

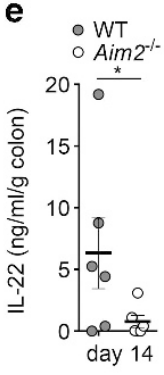

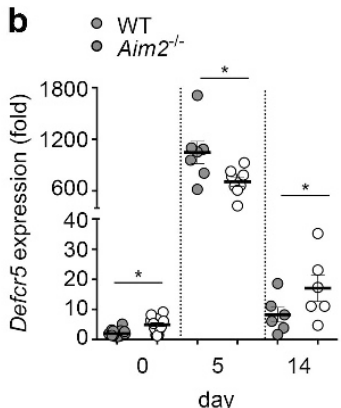

C $:$ WT

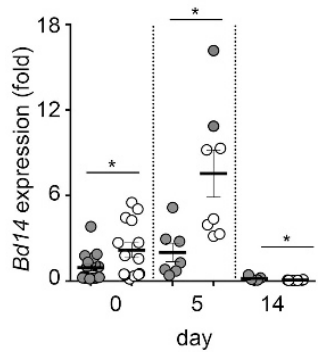

day

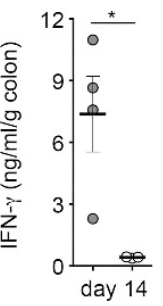

Figure 6 Loss of Aim2 promotes Reg3 $/ \gamma$ production and improved recovery from colitis. Colitis was induced in WT and Aim2-1- mice with $3 \%$ DSS. (a-d) IECs were isolated at days 0, 5 and 14 after DSS administration and were analyzed by real-time PCR for the expression of (a) Reg3b and Reg3g, (b) Defcr5 and (c) Bd14 and (d) I/22bp ( $n=7-16)$. The data are presented as fold induction compared with naive WT mice shown in Figure 5. (e) Spontaneous release of IL-22, TNF- $\alpha$, IL- $1 \beta$ and IFN- $\gamma$ was measured by ELISA from cleared culture supernatants obtained after ex vivo culture for $17 \mathrm{~h}$ of colonic tissue explants obtained 14 days after DSS administration. ( $n=4-5)$, and error bars represent \pm s.e.m.; ${ }^{*} P<0.05$.

time, IL-18 could not have modulated IL-22 through the regulation of IL-22BP, as shown in Figure 6d. Reg3 expression requires STAT3 activation. ${ }^{72,73}$ Therefore, we investigated the expression of Reg3b and Reg $3 g$ proteins in isolated IECs, which produce IL-18 but not IL-22. Expression of Reg3 members was dependent on IL-18 because Reg $3 b$ and Reg3g expression was diminished in the presence of an IL-18 neutralizing antibody (Figure 7e). In addition, Reg3b and Reg3g expression was completely abrogated in Aim2 $2^{-l-}$ and $A s c^{-l-}$ IECs (Figure 7e), indicating that their expression is dependent on AIM2 inflammasome-mediated IL-18 release.

Because IECs do not produce IL-22, the AIM2 inflammasome likely contributes to Reg3b and Reg3g expression through IL-18 and IL-22 by both STAT3-dependent and -independent mechanisms. To confirm this hypothesis and to directly demonstrate the contribution of IL-18 and/or IL-22 to the regulation of Reg3b and Reg3g expression in IECs, we stimulated WT IECs with recombinant IL-18 and IL-22 as above and determined the expression of Reg3b and Reg3g by qPCR. Both IL-18 (Figure 7f) and IL-22 (Figure 7g) increased Reg $3 b$ and Reg3g expression after $17 \mathrm{~h}$, but they had a more potent effect on Reg3g, indicating that both IL-18 and IL-22 can directly induce expression of Reg3b and $\operatorname{Reg} 3 g$ in IECs.

While the elevated IL-22 during acute colitis was likely sufficient for the enhanced STAT3 activation in Aim2 $2^{-1-}$ colon tissue, it could not explain the sustained STAT3 activation upon resolution of the disease, as the IL-22 levels were significantly lower in Aim2 ${ }^{-I-}$ colonic tissue. Reg3 proteins are known to have both antimicrobial and proliferative properties, and Reg3 $\beta$ promotes the development of pancreatic tumors at least in part through effects on STAT3 signaling. ${ }^{74}$ We therefore investigated whether the elevated IL-18/IL-22/STAT3-driven expression of Reg3b and Reg3g observed in Aim2 $2^{-1-}$ IECs could in turn further amplify STAT3-driven responses. Incubation of IECs with both recombinant Reg3 $\beta$ and Reg $3 \gamma$ induced phosphorylation of STAT3 on $\mathrm{Tyr}^{705}$ and Akt on $\mathrm{Ser}^{473}$ as determined by immunoblot (Figure 7h), both of which promote cell proliferation and tissue repair. Thus, elevated expression of Reg $3 \beta$ and Reg3 $\gamma$ in Aim2 $2^{-l-}$ IECs contributed to sustained STAT3 activation during the resolution of intestinal inflammation and promoted cellular proliferation via activation of the Akt pathway in IECs. Indeed, such an improved repair is evident from body weight gain during the resolution phase of colitis, which started at day 9 in WT mice and day 10 in $A i m 2^{-1-}$ mice. A linear regression analysis revealed that the slope $(b)$ of the recovery in Aim2 $2^{-1-}$ mice was $74 \%$ increased compared with WT mice (Figure 7i). We therefore directly analyzed cell proliferation in colonic lamina propria tissue sections by Ki-67 staining. We observed no significant difference in proliferation on day 0 . However, during acute colitis on day 5, the lamina propria of Aim2 $2^{-1-}$ mice still contained a larger number of $\mathrm{Ki}-67^{+}$ proliferative cells compared with WT tissue, although this normalized during resolution at day 14 (Figures $7 \mathrm{j}$ and $\mathrm{k}$ ), indicating that AIM2 controls intestinal repair through sustained cell proliferation. 

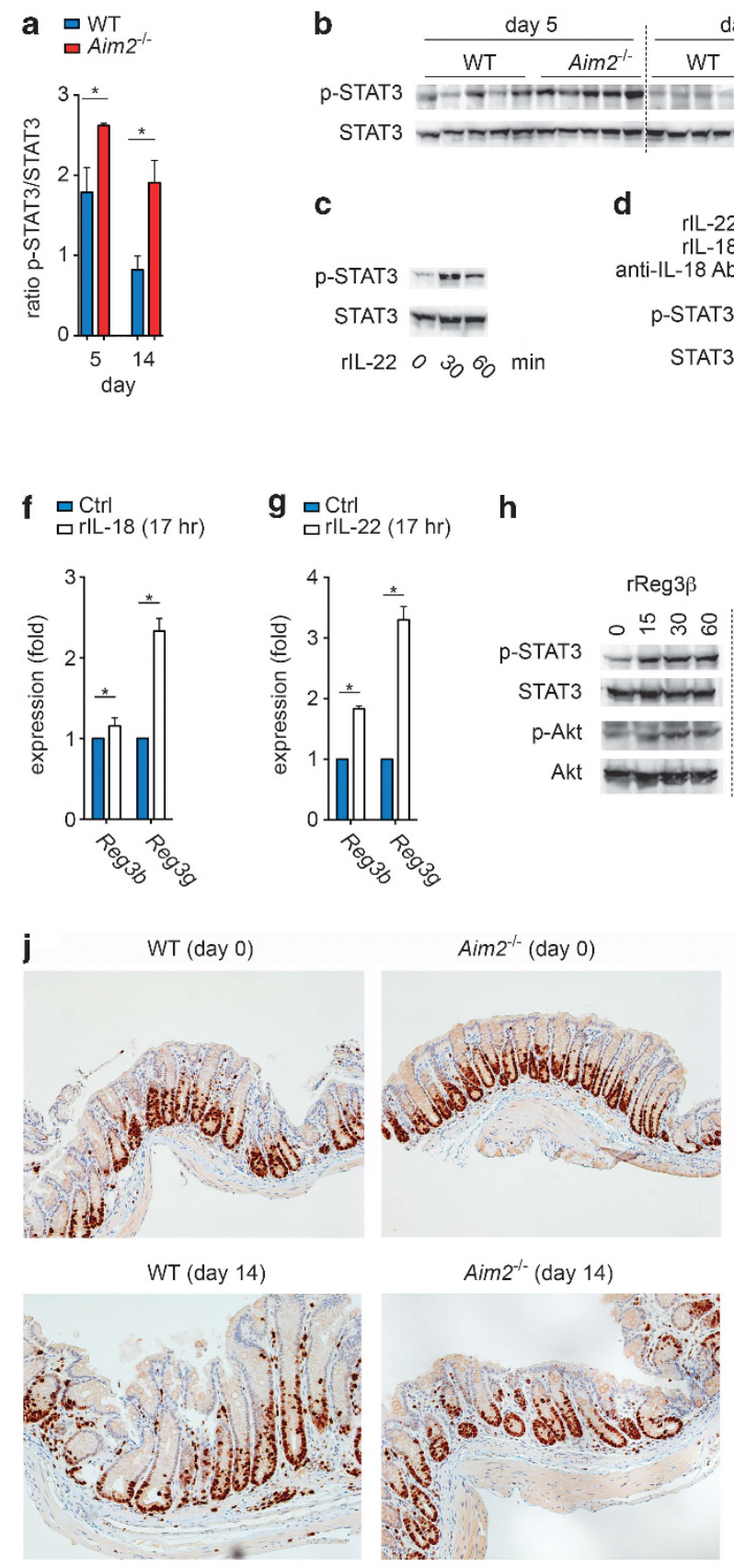

b

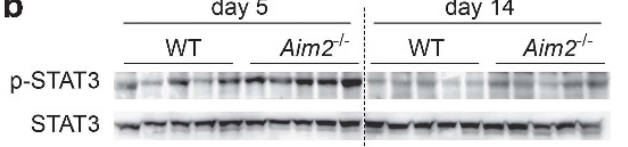

c

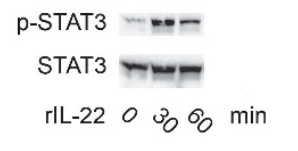

h

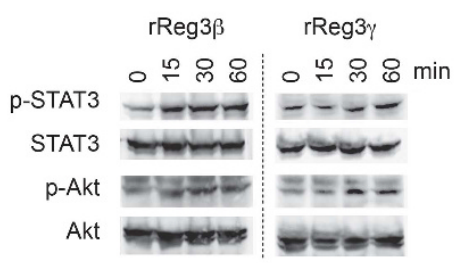

e i

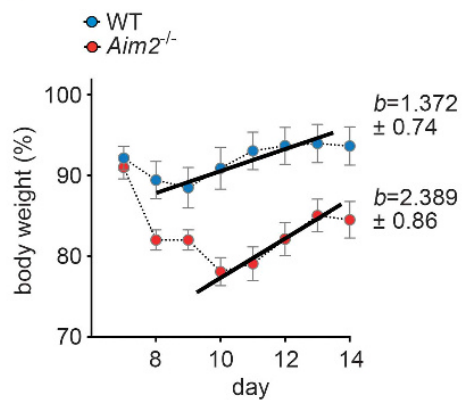

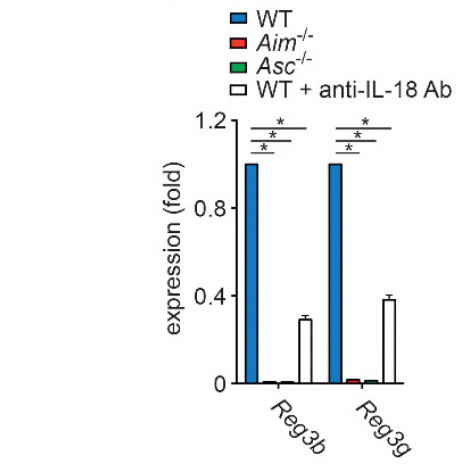

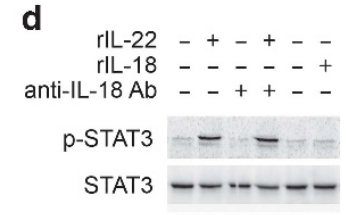


In summary, we demonstrated that the AIM2 inflammasome has a key role in maintaining intestinal homeostasis by promoting adequate expression of AMPs in IECs. As a consequence, loss of Aim2 resulted in transferrable dysbiosis, which rendered Aim2 $2^{-1-}$ mice more susceptible to DSS-induced colitis and promoted excessive and sustained STAT3 activity in IECs through IL-22 signaling and Reg3 expression. This Reg3 $\beta / \gamma$-mediated amplification mechanism contributed to the subsequent improved recovery of $A i m 2^{-1-}$ mice by promoting proliferation and provides a mechanism for the recently observed higher susceptibility of Aim2 $2^{-1-}$ mice to colorectal cancer. ${ }^{29,30}$

\section{DISCUSSION}

IBD is a highly complex disease involving genetic and environmental factors. Several inflammasomes in IECs protect from colitis and colitis-associated colorectal cancer. ${ }^{15,20-25,28}$ In addition, AIM2 protects against colorectal cancer, but this mechanism has been shown to be independent of the AIM2 inflammasome. ${ }^{29,30}$ However, we demonstrated that the AIM2 inflammasome provides a key protective mechanism that prevents dysbiosis-mediated colitis. The AIM2 inflammasome was at least partially responsible for producing adequate levels of IL-18 to maintain well-balanced expression of intestinal AMPs. AIM2 is activated by cytosolic double-stranded DNA during viral and bacterial infection ${ }^{33,61,62}$ and has consequently been linked to self-DNA-driven autoimmunity. ${ }^{75-77}$

Here we demonstrated that AIM2 is activated in IECs in response to double-stranded DNA isolated from fecal microbiota and that this response mediates the release of IL-18 but not IL- $1 \beta$ in IECs, while macrophages respond with the release of both cytokines in an AIM2 inflammasome-dependent manner. Hence, IL-18 production was significantly reduced in Aim2 $2^{-1-}$ mice. Local inflammasome-mediated IL-18 production in the intestine is required for maintaining intestinal health by suppressing dysbiosis from colitogenic microbiota species. ${ }^{15}$ In agreement with the importance of IL-18 in preventing dysbiosis, we observed that AIM2 inflammasome-deficient mice were more susceptible to DSS-induced colitis, suggesting that DSSinduced injury sensitizes the gut to microbiome-derived DNA and effectively induces IL-18 production through activation of the AIM2 inflammasome in the colon, reminiscent of several other inflammasome sensors. Global deletion of inflammasome sensors and effectors has yielded conflicting results in DSS-colitis models. However, the current understanding is that inflammasome activation in infiltrating myeloid cells is detrimental during colitis, primarily through excessive IL-1 $\beta$ production, but inflammasome activation in IECs and the resulting release of IL-18 is necessary for the prevention of dysbiosis and colitis. In addition, the housing of mice determines the composition of the intestinal microbiota and therefore contributes to the susceptibility of mice to DSS-induced colitis and likely also to the relative contribution of a particular inflammasome. We therefore investigated the DSS response in previously analyzed inflammasome-deficient mice, including $\mathrm{Asc}^{-/-}$and Casp $1 / 11^{-/-}$ mice. These mice showed a similar heightened susceptibility to DSS as previously reported, ${ }^{20-22}$ suggesting that the higher susceptibility to DSS-induced colitis in $A i m 2^{-/-}$mice was not solely caused by our housing environment. As expected from the acute inflammation during early colitis, we observed elevated levels of TNF- $\alpha$, IL- $1 \beta$ and IL-22, which were reduced during the resolution phase in Aim $2^{-1-}$ colons, suggesting the existence of improved repair mechanisms in Aim2 ${ }^{-1-}$ mice; this was further supported by enhanced $\operatorname{Reg} 3 b$ and $\operatorname{Reg} 3 g$ expression. The increased production of IL-1 $\beta$ was not surprising, as the hyper-inflammatory environment in the colons of Aim $2^{-1-}$ mice was reflected by increased leukocyte infiltration as well as the participation of several other inflammasomes in IL-1 $\beta$ production during colitis. ${ }^{3,10}$ Hence, loss of only Aim2 did not prevent other inflammasomes from producing this cytokine, which is in agreement with another recent study that observed elevated IL-1 $\beta$ levels in colon tissue of $A i m 2^{-1-}$ mice during the development of colorectal cancer. ${ }^{30}$

To further investigate the underlying mechanism by which the AIM2 inflammasome protected from colitis, we focused on signaling downstream of IL-18 in IECs based on the impaired IL-18 release from Aim2 $2^{-/-}$colons. IL-22 is a key cytokine produced during colitis by subsets of $\mathrm{CD} 4^{+} \mathrm{T}$ cells and ILC3s, and its soluble inhibitory receptor, IL-22BP, is downregulated by IL-18 produced by the NLRP3 and NLRP6 inflammasomes. ${ }^{63}$ IL-22BP is mainly produced by lamina propria $\mathrm{CD} 103^{+} \mathrm{CD} 11 \mathrm{~b}^{+}$ DCs and to a lesser extent by IECs. ${ }^{45}$ IL-22BP and IL-22 are reciprocally expressed during colitis, with IL-22BP being suppressed and IL-22 upregulated to enable enhanced IL-22 signaling in the inflamed intestine, especially in the IECs. The central role of IL-22 in the immunity of superficial tissues has recently emerged, and IL-22 has opposing roles during the progression of colitis. IL-22 exacerbates inflammation during the acute phase, while it promotes tissue repair during the resolution phase, which can lead to colorectal cancer. ${ }^{64}$ In agreement with impaired IL-18 production in Aim2 ${ }^{-1-}$ IECs, we observed enhanced production and release of IL-22BP, which prevented appropriate IL-22 signaling in naive mice. During acute colitis, both WT and Aim2 $2^{-1-}$ IECs showed significant reductions of IL22bp, yet we observed elevated IL-22 levels and increased phosphorylation of STAT3 on $\mathrm{Tyr}^{705}$ in Aim2 $2^{-1-}$ colonic tissue. Hence, derailed IL-18 and IL-22BP during colitis was not sufficient to dampen the increased intestinal IL-22 levels. IL-22 signals preferentially through STAT3, and we also observed increased phosphorylation of STAT3 on $\mathrm{Tyr}^{705}$ in IECs directly activated with recombinant IL-22, but not with IL-18, even when testing up to 50-fold higher IL-18 concentrations.

In addition to its pro-inflammatory and tissue repair functions, IL-22 also has a key anti-microbial role. ${ }^{64}$ Hence, the impaired IL-22 signaling likely affected intestinal homeostasis, and overwhelming evidence links dysbiosis to the pathology of IBD and colitis. ${ }^{52}$ Indeed, naive Aim $2^{-1-}$ mice, even at a young age, already displayed dysbiosis of select bacterial groups. In particular, we detected increased populations of the phylum Bacteroidetes, which are mutualistic Gram-negative, obligate anaerobic, non-endospore-forming bacilli that represent the most substantial portion of the microbiota. Species within the genus Prevotella were particularly increased, and these are frequently recovered from anaerobic infections and are also 
more abundant in $\mathrm{Nlrp6}^{-1-}$ and $A s \mathrm{c}^{-1-}$ mice. ${ }^{15}$ However, we detected no changes in several other bacterial groups. In contrast to $N \operatorname{lrp6^{-1-}}$ and $A s c^{-1-}$ mice, which show increased $T M 7,{ }^{15}$ we observed reduced TM7 numbers in Aim2 $2^{-1-}$ mice, indicating that multiple inflammasomes within IECs collaborate to prevent dysbiosis.

Appropriate antimicrobial barriers and intestinal homeostasis, which are lost during colitis, are maintained by AMPs, which are primarily produced by IECs. ${ }^{67,70}$ In agreement with altered luminal microbiota, naive Aim $2^{-1-}$ mice displayed dysregulated expression of several key AMPs. In particular, expression of both murine Reg3 members Reg $3 b$ and Reg3g was downregulated in Aim $2^{-1-}$ mice. Surprisingly, not all AMPs were lost, and several were even expressed at increased levels in $A i m 2^{-1-}$ mice, including Defcr5, Bd14 and Reg2, while others were expressed at levels equal to WT mice. Elevated expression of some AMPs may potentially constitute a compensatory mechanism to mitigate the loss of Reg3 members. Alternatively, the selective loss of Reg 3 members may explain the amplification of only some bacterial groups in Aim2 $2^{-/-}$mice. AIM2 was therefore required for balanced AMP expression in IECs. AIM2 also collaborates with other inflammasomes, as dysregulated AMP expression is also observed in Nlrp $3^{-1-}$ mice. ${ }^{22}$ IL-18 and IL-22 have both been linked to the expression of AMPs in IECs, ${ }^{68,69}$ and IL-22 in particular is linked to the expression of $\operatorname{Reg} 3$ members. ${ }^{71}$ The IL-22 downstream signaling component STAT3 has an innate antibacterial role in the lung and protects against MRSA pneumonia through the induction of Reg3g. ${ }^{72}$ Therefore, the impaired IL-18 and IL-22 signaling in Aim2 ${ }^{-1-}$ mice could explain the loss of Reg3b and Reg3g, and we directly demonstrated that both cytokines were sufficient for Reg3b and Reg3g expression in IECs. Impaired IL-18 and IL-22 signaling and the resulting defect in AMP expression in Aim2 $2^{-1-}$ IECs consequently promoted dysbiosis and ultimately increased the susceptibility of Aim $2^{-/-}$mice to colitis. Indeed, by fecal microbiota transplant and antibiotic treatment experiments, we directly demonstrated that a transferrable dysbiosis was responsible for the susceptibility to colitis in Aim $2^{-1-}$ mice. Hence, AIM2 has a key role in maintaining intestinal homeostasis. Excessive IL-22 signaling and downstream STAT3 activation during acute colitis further exacerbated inflammation in Aim $2^{-/-}$mice. However, while STAT3 activity was markedly reduced during the resolution phase at day 14 after DSS administration, it was still significantly elevated in Aim2 $2^{-1-}$ colons. This was surprising, as we detected lower IL-22 levels than in WT mice, suggesting that IL-22 alone was likely not sufficient for the elevated STAT3 signaling. Therefore, Aim 2 deficiency also affected other STAT3activating pathways or pathways that sustained IL-22-mediated activities.

While phosphorylation of STAT3 has been identified in IBD patients and in mice, where it promotes acute intestinal inflammation, ${ }^{78,79}$ IEC STAT3 also has an important role in wound healing and tissue repair during the resolution of DSSinduced colitis by promoting IEC survival and proliferation, a repair mechanism that if left unchecked results in increased susceptibility to colorectal cancer. ${ }^{56,80,81}$ Furthermore, STAT3 regulates Reg3 expression, pSTAT3 ${ }^{+}$Th17-cell activation and IL-17 production; accordingly, conditional deletion of Stat3 in IECs exacerbates intestinal inflammation. ${ }^{73}$ Therefore, the increased IL-22 signaling present in Aim $2^{-l-}$ colon tissues was likely responsible for the elevated expression of Reg $3 b$ and $R e g 3 g$ through STAT3-mediated signaling during acute colitis. In turn, Reg3 proteins further amplified and sustained STAT3 activation in IECs after IL-22 levels had decreased during intestinal repair. Hence, our findings suggest that Reg3 proteins themselves are responsible for the sustained STAT3 activation in IECs, even after acute colitis has been resolved in a positive feed-forward loop. In addition to STAT3 activation, Reg3 proteins also promote Akt phosphorylation in IECs. While Aim ${ }^{-1-}$ mice were more susceptible to acute intestinal inflammation, the above changes ultimately facilitated enhanced intestinal tissue repair by promoting proliferation. Hence, AIM2 regulates proliferation of intestinal cells at the base of crypts, which contain Ki-67 $7^{+}$intestinal stem cells and other progenitors, including the Delta-like $1^{+}\left(\mathrm{Dll1} 1^{+}\right)$cells, which are the common precursors of the Paneth, goblet and the enteroendocrine cells, and the secretory label-retaining cells (LRCs), which differentiate into Paneth and enteroendocrine cells. ${ }^{82-84}$ As a chemoattractant for macrophages, Reg $3 \beta$ also promotes tissue repair ${ }^{42}$ and M2 macrophage differentiation. ${ }^{74}$ Hence, it is feasible that elevated Reg3 $\beta$ may be responsible for recruiting M2 macrophages into the inflamed colon to initiate intestinal repair, a hypothesis that is supported by the reduced pro-inflammatory M1-type cytokines TNF- $\alpha$, IL- $1 \beta$ and IFN- $\gamma$ in Aim $2^{-/-}$colon tissue 14 days after DSS administration. This finding is of importance, as two recent studies demonstrated that Aim $2^{-l-}$ mice are more susceptible to induced and spontaneous forms of colorectal cancer. ${ }^{29,30}$ Although an inflammasome-independent mechanism has been suggested based on unchanged IL- $1 \beta$ release, Aim 2 -I- mice show increased proliferation of enterocytes and intestinal stem cells through aberrant Akt activation and expression of proliferative genes, but not Reg3b or Reg3g. ${ }^{29,30}$ AIM2 also interacts and inhibits the PI3K family member DNAPK upstream of Akt. ${ }^{30}$ Aim $2^{-1-}$ mice display dysbiosis, although it involves different bacterial species than those detected in our study, such as reduced Prevotella, indicating housing differences likely have a role in the composition of the microbiota in Aim2 ${ }^{-1-}$ mice. $^{29}$ During the preparation of this manuscript, Zaki et al. also demonstrated AIM2 inflammasome-mediated control of dysbiosis, AMPs and colitis, but they also identified different bacteria amplified in Aim2 $2^{-1-}$ mice, such as Escherichia coli, which were not affected in our study. ${ }^{85}$

In summary, our findings provide important insights into the mechanisms by which AIM2 maintains intestinal homeostasis and prevents colitis and colorectal cancer by demonstrating that AIM2-mediated IL-18 production is a key step in preventing intestinal inflammation. Consequently, loss of Aim2 results in dysregulated IL-18-dependent IL-22BP/IL-22 equilibrium, which is required for balanced AMP expression by IECs and results in the loss of Reg3b and Reg3g expression and dysbiosis in naive mice. Dysbiosis increases the susceptibility of Aim2 ${ }^{-1-}$ mice to experimental colitis in response to damage by DSS, which 


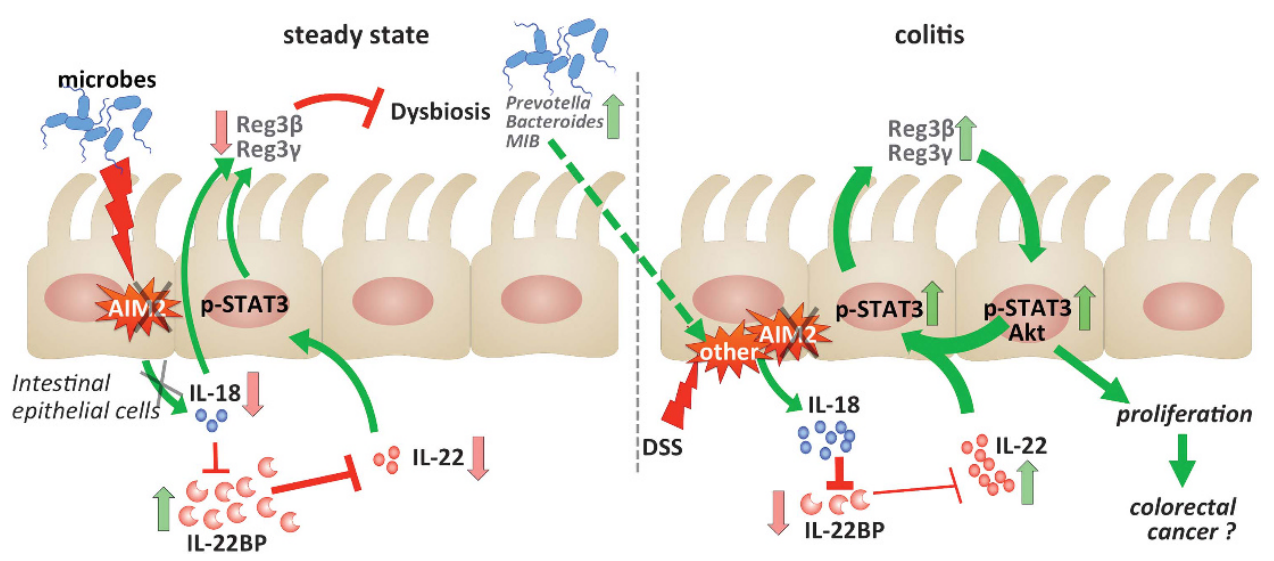

Figure 8 Our model depicting the role of the AIM2 inflammasome in maintaining intestinal homeostasis and how loss of Aim2 promotes dysbiosis and colitis. AIM2 promotes IL-18 production in response to microbial DNA in IECs, which downregulates the expression of I/22bp and consequently enhances IL-22 activity in the colon. This is required for a balanced expression of antimicrobial peptides, including Reg3 $\beta$ and Reg3 $\gamma$, to prevent dysbiosis. In addition, IL-18 is capable of positively regulating Reg3 $\beta$ and Reg3 $\gamma$ expression. Conversely, loss of Aim2 impairs IL-18 production, enhances IL-22BP and consequently impairs IL-22, and Reg3 $\beta$ and Reg3 $\gamma$ expression, resulting in dysbiosis and heightened susceptibility to colitis. During colitis, IL-18 production is enhanced, which downregulates IL-22BP expression and amplifies IL-22-mediated STAT3 activation to promote Reg3 $\beta$ and Reg3 $\gamma$ production. In turn, Reg3 $\beta$ and Reg3 $\gamma$ further promote and sustain STAT3 and Akt activation in a self-amplifying feed-forward loop, which promotes proliferation of crypt cells and intestinal repair. Loss of Aim2 dysregulates this signaling axis, resulting in improved proliferation and repair during the resolution of colitis, which may eventually lead to colorectal cancer.

further dysregulates IL-22 secretion and consequently induces Reg $3 b$ and Reg $3 g$ expression in Aim $2^{-1-}$ mice. Reg3 $\beta$ and Reg3 $\gamma$ perpetuate STAT3 and Akt activation in a forward amplification loop to sustain IEC proliferation and tissue repair, which may contribute to the previously reported heightened susceptibility to colorectal cancer (Figure 8). 29,30,86

\section{CONCLUSIONS}

In the present study, we demonstrate that the AIM2 inflammasome is activated in intestinal epithelial cells by fecal microbiota DNA as a protective mechanism to prevent dysbiosis and colitis, and to regulate intestinal tissue repair through the IL-18/IL-22/ STAT3/Reg3 pathway.

\section{CONFLICT OF INTEREST}

The authors declare no conflict of interest.

\section{ACKNOWLEDGEMENTS}

This work was supported by the National Institutes of Health (AI099009, AI120618 to CS, AR066739 to AD, and a Cancer Center Support Grant CA060553) and the American Heart Association (15POST25690052) to MI Pycard ${ }^{-1-}$ and Nlrp3 $3^{-1-}$ mice were kindly provided by Vishva M. Dixit (Genentech). This work was supported by the Northwestern University Mouse Histology and Phenotyping Laboratory.

1 Ordás I, Eckmann L, Talamini M, Baumgart DC, Sandborn WJ. Ulcerative colitis. Lancet 2012; 380: 1606-1619.

2 Baumgart DC, Sandborn WJ. Crohn's disease. Lancet 2012; 380: 1590-1605.

3 Aguilera M, Darby T, Melgar S. The complex role of inflammasomes in the pathogenesis of inflammatory bowel diseases - lessons learned from experimental models. Cytokine Growth Factor Rev 2014; 25: 715-730.
4 Bergstrom KS, Sham HP, Zarepour M, Vallance BA. Innate host responses to enteric bacterial pathogens: a balancing act between resistance and tolerance. Cell Microbiol 2012; 14: 475-484.

5 Schroder K, Tschopp J. The inflammasomes. Cell 2010; 140: 821-832.

6 Ratsimandresy RA, Dorfleutner A, Stehlik C. An update on PYRIN domain-containing pattern recognition receptors: from immunity to pathology. Front Immunol 2013; 4: 440.

7 Parlato M, Yeretssian G. NOD-like receptors in intestinal homeostasis and epithelial tissue repair. Int J Mol Sci 2014; 15: 9594-9627.

8 Franchi L, Muñoz-Planillo R, Núñez G. Sensing and reacting to microbes through the inflammasomes. Nat Immunol 2012; 13: 325-332.

9 von Moltke J, Ayres JS, Kofoed EM, Chavarría-Smith J, Vance RE. Recognition of bacteria by inflammasomes. Annu Rev Immunol 2013; 31: 73-106.

10 Elinav E, Henao-Mejia J, Flavell RA. Integrative inflammasome activity in the regulation of intestinal mucosal immune responses. Mucosal Immunol 2013; 6: 4-13.

11 Ishikura T, Kanai T, Uraushihara K, liyama R, Makita S, Totsuka T et al. Interleukin-18 overproduction exacerbates the development of colitis with markedly infiltrated macrophages in interleukin-18 transgenic mice. J Gastroenterol Hepatol 2003; 18: 960-969.

12 Kanai T, Watanabe M, Okazawa A, Sato T, Yamazaki M, Okamoto S et al. Macrophage-derived IL-18-mediated intestinal inflammation in the murine model of crohn's disease. Gastroenterology 2001; 121: 875-888.

13 Siegmund B, Fantuzzi G, Rieder F, Gamboni-Robertson F, Lehr HA, Hartmann $\mathrm{G}$ et al. Neutralization of interleukin-18 reduces severity in murine colitis and intestinal ifn-gamma and tnf-alpha production. Am J Physiol Regul Integr Comp Physiol 2001; 281: R1264-R1273.

14 Takagi H, Kanai T, Okazawa A, Kishi Y, Sato T, Takaishi H et al. Contrasting action of IL-12 and IL-18 in the development of dextran sodium sulphate colitis in mice. Scand J Gastroenterol 2003; 38: 837-844.

15 Elinav E, Strowig T, Kau AL, Henao-Mejia J, Thaiss CA, Booth CJ et al. NLRP6 inflammasome regulates colonic microbial ecology and risk for colitis. Cell 2011; 145: 745-757.

16 Loher F, Bauer C, Landauer N, Schmall K, Siegmund B, Lehr HA et al. The interleukin-1 beta-converting enzyme inhibitor pralnacasan reduces dextran sulfate sodium-induced murine colitis and $\mathrm{T}$ helper $1 \mathrm{t}$-cell activation. J Pharmacol Exp Ther 2004; 308: 583-590.

17 Bauer C, Loher F, Dauer M, Mayer C, Lehr HA, Schonharting M et al. The ICE inhibitor pralnacasan prevents dss-induced colitis in C57BL/6 mice and suppresses IP-10 mRNA but not Tnf-alpha mRNA expression. Dig Dis Sci 2007; 52: 1642-1652.

18 Dupaul-Chicoine J, Yeretssian G, Doiron K, Bergstrom KSB, Mclntire $\mathrm{CR}$, LeBlanc PM et al. Control of intestinal homeostasis, colitis, and 
colitis-associated colorectal cancer by the inflammatory caspases. Immunity 2010; 32: 367-378.

19 Bauer C, Duewell P, Mayer C, Lehr HA, Fitzgerald KA, Dauer M et al. Colitis induced in mice with dextran sulfate sodium (DSS) is mediated by the NLRP3 inflammasome. Gut 2010; 59: 1192-1199.

20 Zaki MH, Boyd KL, Vogel P, Kastan MB, Lamkanfi M, Kanneganti T-D. The NLRP3 inflammasome protects against loss of epithelial integrity and mortality during experimental colitis. Immunity 2010; 32: 379-391.

21 Allen IC, TeKippe EM, Woodford RMT, Uronis JM, Holl EK, Rogers AB et al. The NLRP3 inflammasome functions as a negative regulator of tumorigenesis during colitis-associated cancer. J Exp Med 2010; 207: $1045-1056$.

22 Hirota SA, Ng J, Lueng A, Khajah M, Parhar K, Li Y et al. NLRP3 inflammasome plays a key role in the regulation of intestinal homeostasis. Inflamm Bowel Dis 2011; 17: 1359-1372.

23 Zaki MH, Vogel P, Body-Malapel M, Lamkanfi M, Kanneganti T-D. IL-18 production downstream of the NIrp3 inflammasome confers protection against colorectal tumor formation. J Immunol 2010; 185: 4912-4920.

24 Chen GY, Liu M, Wang F, Bertin J, Nunez G. A functional role for Nirp6 in intestinal inflammation and tumorigenesis. J Immunol 2011; 186: 7187-7194.

25 Normand S, Delanoye-Crespin A, Bressenot A, Huot L, Grandjean T, Peyrin-Biroulet $\mathrm{L}$ et al. Nod-like receptor pyrin domain-containing protein 6 (NLRP6) controls epithelial self-renewal and colorectal carcinogenesis upon injury. Proc Natl Acad Sci USA 2011; 108: 9601-9606.

26 Allen IC, Wilson JE, Schneider M, Lich JD, Roberts RA, Arthur JC et al. NLRP12 suppresses colon inflammation and tumorigenesis through the negative regulation of noncanonical nf-kb signaling. Immunity 2012; 36: 742-754

27 Zaki MH, Vogel P, Malireddi RKS, Body-Malapel M, Anand PK, Bertin J et al. The nod-like receptor NLRP12 attenuates colon inflammation and tumorigenesis. Cancer Cell 2011; 20: 649-660.

$28 \mathrm{Hu} \mathrm{B}$, Elinav E, Huber S, Booth CJ, Strowig T, Jin C et al. Inflammationinduced tumorigenesis in the colon is regulated by caspase-1 and NLRC4. Proc Natl Acad Sci USA 2010; 107: 21635-21640.

29 Man SM, Zhu Q, Zhu L, Liu Z, Karki R, Malik A et al. Critical role for the DNA sensor AIM2 in stem cell proliferation and cancer. Cell 2015; 162: 45-58.

30 Wilson JE, Petrucelli AS, Chen L, Koblansky AA, Truax AD, Oyama Y et al. Inflammasome-independent role of AIM2 in suppressing colon tumorigenesis via DNA-PK and akt. Nat Med 2015; 21: 906-913.

31 Mariathasan S, Newton K, Monack DM, Vucic D, French DM, Lee WP et al. Differential activation of the inflammasome by caspase-1 adaptors ASC and ipaf. Nature 2004; 430: 213-218.

32 Mariathasan S, Weiss DS, Newton K, McBride J, O'Rourke K, RooseGirma $\mathrm{M}$ et al. Cryopyrin activates the inflammasome in response to toxins and ATP. Nature 2006; 440: 228-232.

33 Rathinam VA, Jiang Z, Waggoner SN, Sharma S, Cole LE, Waggoner L et al. The AIM2 inflammasome is essential for host defense against cytosolic bacteria and DNA viruses. Nat Immunol 2010; 11: 395-402.

34 Kuida K, Lippke JA, Ku G, Harding MW, Livingston DJ, Su MS et al. Altered cytokine export and apoptosis in mice deficient in interleukin-1 beta converting enzyme. Science 1995; 267: 2000-2003.

35 Khare S, Ratsimandresy RA, de Almeida L, Cuda CM, Rellick SL, Misharin AV et al. The PYRIN domain-only protein POP3 inhibits ALR inflammasomes and regulates responses to infection with DNA viruses. Nat Immunol 2014; 15: 343-353.

36 de Almeida L, Khare S, Misharin AV, Patel R, Ratsimandresy RA, Wallin $\mathrm{MC}$ et al. The PYRIN domain-only protein POP1 inhibits inflammasome assembly and ameliorates inflammatory disease. Immunity 2015; 43: 264-276.

37 Wirtz S, Neufert C, Weigmann B, Neurath MF. Chemically induced mouse models of intestinal inflammation. Nat Protoc 2007; 2: 541-546.

38 Khare S, Dorfleutner A, Bryan NB, Yun C, Radian AD, de Almeida L et al. An nlrp7-containing inflammasome mediates recognition of microbial lipopeptides in human macrophages. Immunity 2012; 36: 464-476.

39 Steinbrecher KA, Harmel-Laws E, Sitcheran R, Baldwin AS. Loss of epithelial rela results in deregulated intestinal proliferative/apoptotic homeostasis and susceptibility to inflammation. J Immunol 2008; 180: 2588-2599.

40 Robertson SJ, Zhou JY, Geddes K, Rubino SJ, Cho JH, Girardin SE et al. Nod1 and nod2 signaling does not alter the composition of intestinal bacterial communities at homeostasis. Gut Microbes 2013; 4: 222-231.

41 Sørensen OE, Thapa DR, Roupé KM, Valore EV, Sjöbring U, Roberts AA et al. Injury-induced innate immune response in human skin mediated by transactivation of the epidermal growth factor receptor. J Clin Invest 2006; 116: 1878-1885.

42 Lörchner H, Pöling J, Gajawada P, Hou Y, Polyakova V, Kostin S et al. Myocardial healing requires reg3 $\beta$-dependent accumulation of macrophages in the ischemic heart. Nat Med 2015; 21: 353-362.

43 Tebar LA, Géranton SM, Parsons-Perez C, Fisher AS, Bayne R, Smith AJH et al. Deletion of the mouse regiiibeta (reg2) gene disrupts ciliary neurotrophic factor signaling and delays myelination of mouse cranial motor neurons. Proc Natl Acad Sci USA 2008; 105: 11400-11405.

44 Zheng Y, Valdez PA, Danilenko DM, Hu Y, Sa SM, Gong Q et al. Interleukin-22 mediates early host defense against attaching and effacing bacterial pathogens. Nat Med 2008; 14: 282-289.

45 Martin JCJ, Bériou G, Heslan M, Chauvin C, Utriainen L, Aumeunier A et al. Interleukin-22 binding protein (IL-22BP) is constitutively expressed by a subset of conventional dendritic cells and is strongly induced by retinoic acid. Mucosal Immunol 2014; 7: 101-113.

46 Barman M, Unold D, Shifley K, Amir E, Hung K, Bos N et al. Enteric salmonellosis disrupts the microbial ecology of the murine gastrointestinal tract. Infect Immun 2008; 76: 907-915.

47 Petnicki-Ocwieja T, Hrncir T, Liu Y-J, Biswas A, Hudcovic T, TlaskalovaHogenova $\mathrm{H}$ et al. Nod2 is required for the regulation of commensal microbiota in the intestine. Proc Natl Acad Sci USA 2009; 106: $15813-15818$.

48 Furet J-P, Firmesse O, Gourmelon M, Bridonneau C, Tap J, Mondot S et al. Comparative assessment of human and farm animal faecal microbiota using real-time quantitative PCR. FEMS Microbiol Ecol 2009; 68: 351-362.

49 Sizova MV, Doerfert SN, Gavrish E, Epstein SS. TM7 detection in human microbiome: Are PCR primers and FISH probes specific enough? J Microbiol Methods 2015; 114: 51-53.

50 Booth C, O'Shea JA. Isolation and culture of intestinal epithelial cells. Culture of Epithelial Cells, Second Edition, vol 10; 2002. pp 303-335.

51 Palleroni AV, Varesio L, Wright RB, Brunda MJ. Tumoricidal alveolar macrophage and tumor infiltrating macrophage cell lines. Int J Cancer 1991; 49: 296-302.

52 Manichanh C, Borruel N, Casellas F, Guarner F. The gut microbiota in IBD. Nat Rev Gastroenterol Hepatol 2012; 9: 599-608.

53 Kitajima S, Takuma S, Morimoto M. Changes in colonic mucosal permeability in mouse colitis induced with dextran sulfate sodium. Exp Anim 1999; 48: 137-143.

54 Okayasu I, Hatakeyama S, Yamada M, Ohkusa T, Inagaki Y, Nakaya R. A novel method in the induction of reliable experimental acute and chronic ulcerative colitis in mice. Gastroenterology 1990; 98: 694-702.

55 Neurath MF. Cytokines in inflammatory bowel disease. Nat Rev Immunol 2014; 14: 329-342.

56 Grivennikov S, Karin E, Terzic J, Mucida D, Yu G-Y, Vallabhapurapu S et al. IL-6 and stat3 are required for survival of intestinal epithelial cells and development of colitis-associated cancer. Cancer Cell 2009; 15: 103-113.

57 Naito Y, Takagi T, Uchiyama K, Kuroda M, Kokura S, Ichikawa H et al. Reduced intestinal inflammation induced by dextran sodium sulfate in interleukin-6-deficient mice. Int J Mol Med 2004; 14: 191-196.

58 Choi JS, Kim K-H, Lau LF. The matricellular protein CCN1 promotes mucosal healing in murine colitis through IL-6. Mucosal Immunol 2015; 8: $1285-1296$.

59 Levy M, Thaiss CA, Zeevi D, Dohnalová L, Zilberman-Schapira G, Mahdi JA et al. Microbiota-Modulated metabolites shape the intestinal microenvironment by regulating NLRP6 inflammasome signaling. Cell 2015; 163: 1428-1443.

60 Geem D, Medina-Contreras O, McBride M, Newberry RD, Koni PA, Denning TL. Specific microbiota-induced intestinal th17 differentiation requires $\mathrm{MHC}$ class II but not GALT and mesenteric lymph nodes. J Immunol 2014; 193: 431-438.

61 Fernandes-Alnemri T, Yu JW, Juliana C, Solorzano L, Kang S, Wu J et al. The AIM2 inflammasome is critical for innate immunity to francisella tularensis. Nat Immunol 2010; 11: 385-393.

62 Jones JW, Kayagaki N, Broz P, Henry T, Newton K, O'Rourke K et al. Absent in melanoma 2 is required for innate immune recognition of francisella tularensis. Proc Natl Acad Sci USA 2010; 107: 9771-9776.

63 Huber S, Gagliani N, Zenewicz LA, Huber FJ, Bosurgi L, Hu B et al. $\mathrm{IL}-22 \mathrm{BP}$ is regulated by the inflammasome and modulates tumorigenesis in the intestine. Nature 2012; 491: 259-263. 
64 Dudakov JA, Hanash AM, van den Brink MR. Interleukin-22: Immunobiology and pathology. Annu Rev Immunol 2015; 33: 747-785.

65 Spits H, Artis D, Colonna M, Diefenbach A, Di Santo JP, Eberl G et al. Innate lymphoid cells-a proposal for uniform nomenclature. Nat Rev Immunol 2013; 13: 145-149.

66 McKenzie ANJ, Spits H, Eberl G. Innate lymphoid cells in inflammation and immunity. Immunity 2014; 41: 366-374.

67 Mukherjee S, Hooper LV. Antimicrobial defense of the intestine. Immunity 2015; 42: 28-39.

68 Hill T, Krougly O, Nikoopour E, Bellemore S, Lee-Chan E, Fouser LA et al. The involvement of interleukin-22 in the expression of pancreatic beta cell regenerative reg genes. Cell Regen (Lond) 2013; 2: 2.

69 McDonald V, Pollok RCG, Dhaliwal W, Naik S, Farthing MJG, Bajaj-Elliott M. A potential role for interleukin-18 in inhibition of the development of cryptosporidium parvum. Clin Exp Immunol 2006; 145: 555-562.

70 Ostaff MJ, Stange EF, Wehkamp J. Antimicrobial peptides and gut microbiota in homeostasis and pathology. EMBO Mol Med 2013; 5: 1465-1483.

71 Manta C, Heupel E, Radulovic K, Rossini V, Garbi N, Riedel CU et al. (3) $\mathrm{CR} 1(+)$ macrophages support IL-22 production by innate lymphoid cells during infection with citrobacter rodentium. Mucosal Immunol 2013; 6: 177-188.

72 Choi SM, McAleer JP, Zheng M, Pociask DA, Kaplan MH, Qin S et al. Innate stat3-mediated induction of the antimicrobial protein reg3 $\gamma$ is required for host defense against MRSA pneumonia. J Exp Med 2013; 210: 551-561.

73 Willson TA, Jurickova I, Collins M, Denson LA. Deletion of intestinal epithelial cell STAT3 promotes T-lymphocyte STAT3 activation and chronic colitis following acute dextran sodium sulfate injury in mice. Inflamm Bowel Dis 2013; 19: 512-525.

74 Gironella M, Calvo C, Fernández A, Closa D, lovanna JL, Rosello-Catafau $\mathrm{J}$ et al. Reg3 $\beta$ deficiency impairs pancreatic tumor growth by skewing macrophage polarization. Cancer Res 2013; 73: 5682-5694.

75 Jakobs C, Perner S, Hornung V. AIM2 drives joint inflammation in a selfdna triggered model of chronic polyarthritis. PLOS One 2015; 10: e0131702.

76 Zhang W, Cai Y, Xu W, Yin Z, Gao X, Xiong S. AlM2 facilitates the apoptotic dna-induced systemic lupus erythematosus via arbitrating macrophage functional maturation. J Clin Immunol 2013; 33: 925-937.

77 Baum R, Sharma S, Carpenter S, Li Q-Z, Busto P, Fitzgerald KA et al. Cutting edge: AIM2 and endosomal tirs differentially regulate arthritis and autoantibody production in dnase ii-deficient mice. J Immunol 2015; 194: 873-877.
78 Carey R, Jurickova I, Ballard E, Bonkowski E, Han X, Xu H et al. Activation of an IL-6:STAT3-dependent transcriptome in pediatric-onset inflammatory bowel disease. Inflamm Bowel Dis 2008; 14: 446-457.

79 Mudter J, Weigmann B, Bartsch B, Kiesslich R, Strand D, Galle PR et al. Activation pattern of signal transducers and activators of transcription (STAT) factors in inflammatory bowel diseases. Am J Gastroenterol 2005; 100: 64-72.

80 Bollrath J, Phesse TJ, von Burstin VA, Putoczki T, Bennecke M, Bateman $\mathrm{T}$ et al. Gp130-mediated stat3 activation in enterocytes regulates cell survival and cell-cycle progression during colitisassociated tumorigenesis. Cancer Cell 2009; 15: 91-102.

81 Pickert G, Neufert C, Leppkes M, Zheng Y, Wittkopf N, Warntjen M et al. STAT3 links IL-22 signaling in intestinal epithelial cells to mucosal wound healing. J Exp Med 2009; 206: 1465-1472.

82 Basak O, van de Born M, Korving J, Beumer J, van der Elst S, van Es JH et al. Mapping early fate determination in lgr5+ crypt stem cells using a novel ki67-rfp allele. EMBO J 2014; 33: 2057-2068.

83 Buczacki SJ, Zecchini HI, Nicholson AM, Russell R, Vermeulen L, Kemp $\mathrm{R}$ et al. Intestinal label-retaining cells are secretory precursors expressing lgr5. Nature 2013; 495: 65-69.

84 van Es JH, van Gijn ME, Riccio O, van den Born M, Vooijs M, Begthel H et al. Notch/gamma-secretase inhibition turns proliferative cells in intestinal crypts and adenomas into goblet cells. Nature 2005; 435: 959-963.

85 Hu S, Peng L, Kwak Y-T, Tekippe EM, Pasare C, Malter JS et al. The DNA sensor AIM2 maintains intestinal homeostasis via regulation of epithelial antimicrobial host defense. Cell Rep 2015; 13: 1922-1936.

86 Vonarbourg C, Mortha A, Bui VL, Hernandez PP, Kiss EA, Hoyler T et al. Regulated expression of nuclear receptor roryt confers distinct functional fates to NK cell receptor-expressing roryt(+) innate lymphocytes. Immunity 2010; 33: 736-751.

(c) (i) (5) (2)

This work is licensed under a Creative Commons

Attribution-NonCommercial-ShareAlike

4.0

International License. The images or other third party material in this article are included in the article's Creative Commons license, unless indicated otherwise in the credit line; if the material is not included under the Creative Commons license, users will need to obtain permission from the license holder to reproduce the material. To view a copy of this license, visit http://creativecommons.org/licenses/by-nc-sa/4.0/ 